University of Wollongong

Research Online

Faculty of Engineering and Information

Faculty of Engineering and Information

Sciences - Papers: Part B

Sciences

2020

\title{
Trapdoor Delegation and HIBE from Middle-Product LWE in Standard Model
}

Quoc Huy Le

University of Wollongong, qhl576@uowmail.edu.au

Dung Hoang Duong

University of Wollongong, hduong@uow.edu.au

Willy Susilo

University of Wollongong, wsusilo@uow.edu.au

Josef Pieprzyk

Follow this and additional works at: https://ro.uow.edu.au/eispapers1

Part of the Engineering Commons, and the Science and Technology Studies Commons

Research Online is the open access institutional repository for the University of Wollongong. For further information contact the UOW Library: research-pubs@uow.edu.au 


\title{
Trapdoor Delegation and HIBE from Middle-Product LWE in Standard Model
}

\author{
Abstract \\ (C) 2020, Springer Nature Switzerland AG. At CRYPTO 2017, Roşca, Sakzad, Stehlé and Steinfeld \\ introduced the Middle-Product LWE (MPLWE) assumption which is as secure as Polynomial-LWE for a \\ large class of polynomials, making the corresponding cryptographic schemes more flexible in choosing \\ the underlying polynomial ring in design while still keeping the equivalent efficiency. Recently at TCC \\ 2019, Lombardi, Vaikuntanathan and Vuong introduced a variant of MPLWE assumption and constructed \\ the first IBE scheme based on MPLWE. Their core technique is to construct lattice trapdoors compatible \\ with MPLWE in the same paradigm of Gentry, Peikert and Vaikuntanathan at STOC 2008. However, their \\ method cannot directly offer a Hierarchical IBE construction. In this paper, we make a step further by \\ proposing a novel trapdoor delegation mechanism for an extended family of polynomials from which we \\ construct, for the first time, a Hierachical IBE scheme from MPLWE. Our Hierarchy IBE scheme is provably \\ secure in the standard model. \\ Disciplines \\ Engineering | Science and Technology Studies

\section{Publication Details} \\ Le, H., Duong, H., Susilo, W. \& Pieprzyk, J. (2020). Trapdoor Delegation and HIBE from Middle-Product LWE \\ in Standard Model. Lecture Notes in Computer Science (including subseries Lecture Notes in Artificial \\ Intelligence and Lecture Notes in Bioinformatics), 12146 LNCS 130-149.
}




\title{
Trapdoor Delegation and HIBE from Middle-Product LWE in Standard Model
}

\author{
Huy Quoc Le ${ }^{1,2(\bowtie)}$, Dung Hoang Duong ${ }^{1(\bowtie)}$, Willy Susilo ${ }^{1}$, Josef Pieprzyk ${ }^{2,3}$ \\ 1 Institute of Cybersecurity and Cryptology, School of Computing and Information \\ Technology, University of Wollongong, Northfields Avenue, Wollongong NSW 2522, \\ Australia. \\ qh1576@uowmail.edu.au, \{hduong, wsusilo\}@uow.edu.au \\ 2 CSIRO Data61, Sydney, NSW, Australia. \\ 3 Institute of Computer Science, Polish Academy of Sciences, Warsaw, Poland. \\ josef .pieprzyk@data61.csiro.au
}

\begin{abstract}
At CRYPTO 2017, Roşca, Sakzad, Stehlé and Steinfeld introduced the Middle-Product LWE (MPLWE) assumption which is as secure as Polynomial-LWE for a large class of polynomials, making the corresponding cryptographic schemes more flexible in choosing the underlying polynomial ring in design while still keeping the equivalent efficiency. Recently at TCC 2019, Lombardi, Vaikuntanathan and Vuong introduced a variant of MPLWE assumption and constructed the first IBE scheme based on MPLWE. Their core technique is to construct lattice trapdoors compatible with MPLWE in the same paradigm of Gentry, Peikert and Vaikuntanathan at STOC 2008. However, their method cannot directly offer a Hierachical IBE construction. In this paper, we make a step further by proposing a novel trapdoor delegation mechanism for an extended family of polynomials from which we construct, for the first time, a Hierachical IBE scheme from MPLWE. Our Hierachy IBE scheme is provably secure in the standard model.
\end{abstract}

Key words: Middle-Product LWE, trapdoor, HIBE, standard model, lattices

\section{Introduction}

Hierarchical identity-based encryption (HIBE) [7,9] is a variant of IBE [17], which embeds a directed tree. The nodes of the tree are identities and the children identities are produced by appending extra information to their parent identities. HIBEs can be found in many applications such as forward-secure encryption [3], broadcast encryption $[5,19]$ and access control to pervasive computing information [8] to name a few most popular.

In lattice-based cryptography, a crucial tool for constructing IBE and HIBE schemes is a trapdoor. The GPV construction, for instance, applies trapdoor preimage sampleable functions [6]. The trapdoor plays a role of master secret key that is used to sample private key for each identity (following a distribution that is negligibly close to uniform). This trapdoor is applied by Gentry et al. [6] 
to construct their IBE from lattices in the random oracle model. Using the same paradigm as [6], Agrawal et al. [1] introduced their IBE scheme in the standard model. Cash et al. [4] define bonsai tree with four basic principles in delegating a lattice basis (i.e., delegating a trapdoor in the [6] sense). The bonsai tree technique helps to resolve some open problems in lattice-based cryptography. It allows us to construct some lattice-based primitives in the standard model (without random oracles) as well as it facilitates delegation for purposes such as lattice-based HIBE schemes. At the same time, Agrawal et al. [2] proposed two distinct trapdoor delegations following the definition of trapdoor from [6]. Their techniques have been used to construct a HIBE scheme in the standard model, which is more efficient than the one from [4]. Micciancio and Peikert in their work [13] introduced a simpler and more efficient trapdoor generation and delegation mechanism.

The middle-product learning with errors problem (MPLWE) is a variant of the polynomial learning with error problem (PLWE) proposed by Roşca et al. [16]. It exploits the middle-product of polynomials modulo $q$. The authors of [16] have proved that MPLWE is as secure as PLWE for a large class of polynomials. This allows more flexibility in choosing underlying polynomial rings when designing cryptosystems. In [16], the authors have constructed a Regev-type public key encryption scheme based on MPLWE, which is as efficient as that built over Ring-LWE [12]. Recently, Lombardi et al. [10] have generalized MPLWE and call it degree-parametrized MPLWE (DMPLWE). They have proved that DMPLWE is as hard as PLWE using similar arguments as in [16]. Further, the authors of [10] have introduced a lattice trapdoor construction (following the trapdoor notion of [13]) for DMPLWE. The construction can be used to design a dual Regev encryption. The dual encryption allows the authors of [10] to come up with IBE constructions in both the random oracle model and the standard model. The standard model IBE in [10] is adapted from the framework of [1]. However, a DMPLWE-based construction for a standard model HIBE cannot be directly obtained from the standard model IBE of [10]. Thus there is a need for more work in order to define an appropriate trapdoor delegation mechanism for the polynomial setting.

Our contribution. In this paper, we follow the line of research initiated by the work [10]. In particular, we introduce a novel technique for delegating lattice trapdoors from DMPLWE and construct a new HIBE scheme based on DMPLWE. Our HIBE scheme is provably secure in the standard model. We follow the framework from [1] and [10].

Let $\overline{\mathbf{a}}=\left(a_{1}, \cdots, a_{t^{\prime}}\right)$ be a $t^{\prime}$-family of polynomials. We can interpret any polynomial as a structured matrix, e.g. Toeplitz matrix [15], and hence $\overline{\mathbf{a}}$ can be represented as a concatenated structured matrix, say A. The trapdoor from [10] is a modification of the trapdoor used in [13] and is defined for a family of polynomials. More specifically, in [10], a trapdoor for the family $\overline{\mathbf{a}}$ is a collection $\operatorname{td}_{a}$ of short polynomials (here short means small coefficients), from which we form a matrix $\mathbf{R}$ such that $\mathbf{A} \cdot\left[\begin{array}{c}\mathbf{R} \\ \mathbf{I}\end{array}\right]=\mathbf{G}$, where $\mathbf{G}$ is the concatenated structured matrix of $\overline{\mathbf{g}}=\left(g_{1}, \cdots, g_{\gamma \tau}\right)$, namely $g_{j}=2^{\eta} x^{d \zeta}$ for $j=\zeta \tau+\eta+1$ with $\eta \in$ 
$\{0, \cdots, \tau-1\}, \zeta \in\{0, \cdots, \gamma-1\}$. We call $\mathbf{g}$ the primitive family. The trapdoor $\operatorname{td}_{a}$ is used to search for a $t^{\prime}$-family of polynomials $\overline{\mathbf{r}}:=\left(r_{1}, \cdots, r_{t^{\prime}}\right)$ (following some distribution that is close to uniform) such that $\langle\overline{\mathbf{a}}, \overline{\mathbf{r}}\rangle:=\sum_{i=1}^{t^{\prime}} a_{i} \cdot r_{i}=u$ for any given polynomial $u$ of appropriate degree.

For a construction of DMPLWE-based HIBE, we need to derive a trapdoor for an extended family of polynomials, say $\overline{\mathbf{f}}=(\overline{\mathbf{a}} \mid \overline{\mathbf{h}})=\left(a_{1}, \cdots, a_{t^{\prime}} \mid h_{1}, \cdots, h_{t^{\prime \prime}}\right)$, from a trapdoor for $\overline{\mathbf{a}}$. To this end, we first proceed with the case $t^{\prime \prime}=\gamma \tau$, i.e., the number of polynomials in $\overline{\mathbf{h}}$ has to be the same as the number in $\overline{\mathbf{g}}$. We transform $\overline{\mathbf{h}}$ into a matrix $\mathbf{H}$, and then apply the idea of trapdoor delegation from [13] to obtain the trapdoor $\mathrm{td}_{f}$ for $\overline{\mathbf{f}}$. We generalize the trapdoor delegation to the case $t^{\prime \prime}=m \gamma \tau$, a multiple of $\gamma \tau$ for $m \geq 1$.

Using the proposed polynomial trapdoor delegation, we build the first HIBE based on DMPLWE, which is provably INDr-sID-CPA secure in the standard model. To produce a private key for an identity id $=\left(i d_{1}, \cdots, i d_{\ell}\right)$ at depth $\ell$, we form an extended family $\overline{\mathbf{f}}_{\text {id }}=\left(\overline{\mathbf{a}}, \overline{\mathbf{h}}^{\left(1, i d_{1}\right)}, \cdots, \overline{\mathbf{h}}^{\left(\ell, i d_{\ell}\right)}\right)$ in which each $\overline{\mathbf{h}}^{(i, \text { bit })}=\left(h_{1}^{(i, \text { bit })}, \cdots, h_{t^{\prime}}^{(i, \text { bit })}\right)$ is a family of random polynomials. Then our trapdoor delegation helps to get a trapdoor for $\overline{\mathbf{f}}_{\text {id }}$, which plays the role of the private key with respect to the identity id. Deriving a private key for a child identity id $\mid i d_{\ell+1}=\left(i d_{1}, \cdots, i d_{\ell}, i d_{\ell+1}\right)$ from a parent identity id $=\left(i d_{1}, \cdots, i d_{\ell}\right)$ is done in similar way by appending $\overline{\mathbf{h}}^{\left(\ell+1, i d_{\ell+1}\right)}$ to $\overline{\mathbf{f}}_{\text {id }}$ so we get $\overline{\mathbf{f}}_{\mathrm{id} \mid i d_{\ell+1}}=$ $\left(\overline{\mathbf{a}}, \overline{\mathbf{h}}^{\left(1, i d_{1}\right)}, \cdots, \overline{\mathbf{h}}^{\left(\ell, i d_{\ell}\right)}, \overline{\mathbf{h}}^{\left(\ell+1, i d_{\ell+1}\right)}\right)$. Then we use the trapdoor delegation to get its private key from the private key (trapdoor) of $\overline{\mathbf{f}}_{\mathrm{id}}$. In order for the security proof to work, we need to put a condition on $t^{\prime}$ such that $t^{\prime}$ is a multiple of $\gamma \tau$. Indeed, the condition ensures that the simulator is able to simulate an answer to a private key query of the adversary. The answer is generated using a trapdoor for some $\overline{\mathbf{h}}^{\left(i, i d_{1}\right)}$, both of which are not chosen randomly but produced by a trapdoor generator.

Open problems. Our trapdoor delegation technique is restricted to the relation of the number of polynomials in the primitive family $\overline{\mathbf{g}}$ and the number of polynomials in the extended family (i.e., $t^{\prime \prime}=m \gamma \tau$, a multiple of $\gamma \tau$ ). It would be interesting and might be useful to find a new trapdoor delegation method that could be applied for an arbitrary $t^{\prime \prime} \geq 1$. Moreover, if we had another mechanism that would help to find a trapdoor for $\overline{\mathbf{f}}_{\text {id }}^{\prime}$, where $\overline{\mathbf{f}}_{\text {id }}^{\prime}=$ $\left(\overline{\mathbf{a}},\left\langle\overline{\mathbf{h}}^{\left(1, i d_{1}\right)}, \overline{\mathbf{b}}\right\rangle, \cdots,\left\langle\overline{\mathbf{h}}^{\left(\ell, i d_{\ell}\right)}, \overline{\mathbf{b}}\right\rangle\right)$, given a random $\overline{\mathbf{b}}$ and its trapdoor $\operatorname{td}_{b}$, then we might be able to apply the HIBE framework of [2] to get a smaller ciphertext size than that of our work here. One more question is that whether or not there exists a trapdoor (and delegation) method that does not utilise the Toeplitz representation but applies directly polynomials, with a relevant definition of polynomial trapdoor.

Organisation. In Section 2, we review some related background. The trapdoor delegation mechanism for polynomials in MPLWE setting will be presented in 
Section 3. We will give an MPLWE-based HIBE construction in the standard model in Section 4. Section 5 concludes this work.

\section{Preliminaries}

Notations. We denote by $R^{<n}[x]$ the set of polynomials of degree less than $n$ with coefficients in a commutative ring $R$. We mainly work with the rings of polynomials over $\mathbb{Z}$ such as $\mathbb{Z}[x]$ and $\mathbb{Z}_{q}[x]$. We use italic small letters for polynomials in $R$. For a positive integer $\ell$, [ $\ell]$ stands for the set $\{1,2, \cdots, \ell\}$. The Gram-Schmidt orthogonal matrix of a matrix $\mathbf{A}$ is written as $\tilde{\mathbf{A}}$. We call $\overline{\mathbf{h}}$ an $n$-family (or $n$-vector) of polynomials if $\overline{\mathbf{h}}=\left(h_{1}, \cdots, h_{n}\right)$, where $h_{i}$ 's are polynomials. By $\mathbf{a} \mid \overline{\mathbf{h}}$, we denote a concatenated (or expanded) family, which consists of all ordered polynomials from both $\overline{\mathbf{a}}$ and $\overline{\mathbf{h}}$. For two $n$-families of polynomials $\overline{\mathbf{a}}=\left(a_{1}, \cdots, a_{n}\right)$ and $\overline{\mathbf{r}}=\left(r_{1}, \cdots, c_{n}\right)$, their scalar product is defined as $\langle\overline{\mathbf{a}}, \overline{\mathbf{r}}\rangle:=\sum_{i=1}^{n} a_{i} \cdot r_{i}$. The notation $\mathcal{U}(X)$ stands for the uniform distribution over the set $X$. The Euclidean and sup norms of a vector $\mathbf{u}$ (as well as a matrix) are written as $\|\mathbf{u}\|$ and $\|\mathbf{u}\|_{\infty}$, respectively.

\subsection{IBE and HIBE: Syntax and Security}

Syntax. An IBE system [17] is a tuple of algorithms \{Setup, Extract, Encrypt, Decrypt\}, in which: (1) Setup $\left(1^{n}\right)$ on input a security parameter $1^{n}$, outputs a master public key MPK and a master secret key MSK; (2) Extract(MSK, id) on input the master secret key MSK and an identity id, outputs a private key SK $_{\text {id }}$; (3) Encrypt(MPK, id, $\mu$ ) on input the master public key MPK, an identity id and a message $\mu$, outputs a ciphertext CT; and (4) Decrypt(id, $\mathrm{SK}_{\text {id }}, \mathrm{CT}$ ) on input an identity id and its associated private key $\mathrm{SK}_{\text {id }}$ and a ciphertet $\mathrm{CT}$, outputs a message $\mu$.

A HIBE [7] is a tuple of algorithms \{Setup, Extract, Derive, Encrypt, Decrypt\}, where Setup, Extract, Encrypt, Decrypt are defined in similar way as for IBE. Let $\lambda$ be the maximum depth of identities. An identity at depth $\ell \leq \lambda$ is represented by a binary vector id $=\left(i d_{1}, \cdots, i d_{\ell}\right) \in\{0,1\}^{\ell}$ of dimension $\ell$ and it is considered as the "parent" of the appended id $\mid i d_{\ell+1}=\left(i d_{1}, \cdots, i d_{\ell}, i d_{\ell+1}\right)$. The algorithm Setup $\left(1^{n}, 1^{\lambda}\right)$ needs a slight modification as it accepts both $n$ and $\lambda$ as the input. For the input: private key $\mathrm{SK}_{\mathrm{id}}$ and id|id $\mathrm{id}_{\ell+1}$, the algorithm Derive $\left(\mathrm{SK}_{\mathrm{id}}, \mathrm{id} \mid \mathrm{id}_{\ell+1}\right)$ outputs the private key $\mathrm{SK}_{\mathrm{id} \mid i d_{\ell+1}}$ for the identity id $\mid i d_{\ell+1}$. If we consider the master secret key as the private key for any identity at depth 0 , then Derive has the same function as Extract. $(\mathrm{H}) \mathrm{IBE}$ has to be correct in the following sense:

$$
\operatorname{Pr}\left[\operatorname{Decrypt}\left(\mathrm{id}, \mathrm{SK}_{\text {id }}, \operatorname{Encrypt}(\mathrm{MPK}, \mathrm{id}, \mu)\right)\right]=1-\operatorname{negl}(n),
$$

where the probability is taken over random coin tosses for Setup, Extract, Encrypt, Decrypt (for IBE) and Derive (for HIBE). 
Security. For the purpose of our paper, we present the following security game for INDr-sID-CPA or indistinguishability of ciphertexts under a selective chosenidentity and adaptive chosen-plaintext attack. In the game, the adversary has to announce his target identity at the very beginning. For a security parameter $n$, let $\mathcal{M}_{n}$ and $\mathcal{C}_{n}$ be the plaintext and ciphertext spaces, respectively. The game consists of six phases as follows:

- Initialize: The challenger chooses a maximum depth $\lambda$ and gives it to the adversary. The adversary outputs a target identity id $^{*}=\left(i d_{1}^{*}, \cdots, i d_{k}^{*}\right),(k \leq$ $\lambda)$.

- Setup: The challenger runs $\operatorname{Setup}\left(1^{n}, 1^{\lambda}\right)$ and sends the public parameters MPK to the adversary. The master secret key MSK is kept secret by the challenger.

- Queries 1: The adversary makes private key queries adaptively. The queries are for identities id of the form id $=\left(i d_{1}, \cdots, i d_{m}\right)$ for some $m \leq \lambda$, which are not a prefix of $\mathrm{id}^{*}$. This is to say that $\mathrm{id}_{i} \neq \mathrm{id}_{i}^{*}$ for all $i \in[m]$ and $m \leq k$. The challenger answers the private key query for id by calling the private key extraction algorithm Extract and sends the key to the adversary.

- Challenge:

- Whenever the adversary decides to finish Queries 1, he will output the challenge plaintext $\mu^{*} \in \mathcal{M}_{n}$.

- The challenger chooses a random bit $b \in\{0,1\}$. It computes the challenge ciphertext $C T^{*}$. If $b=0$, it calls the encryption algorithm and gets $\mathrm{CT}^{*} \leftarrow \operatorname{Encrypt}\left(\mathrm{MPK}, \mathrm{id}^{*}, \mu^{*}\right)$. If $b=1$, it chooses a random $C T \in \mathcal{C}_{n}$ so $\mathrm{CT}^{*} \leftarrow C T$. $\mathrm{CT}^{*}$ is then sent to the adversary.

- Queries 2: The adversary makes the private key queries again and the challenger answers the queries as in Queries 1.

- Guess: The adversary outputs a guess $b^{\prime} \in\{0,1\}$ and he wins if $b^{\prime}=b$.

The adversary in the above game is referred to as an INDr-sID-CPA adversary. The advantage of an adversary $\mathcal{A}$ in the game is $\operatorname{Adv}^{\operatorname{HIBE}, \lambda, \mathcal{A}}(n)=\mid \operatorname{Pr}[b=$ $\left.b^{\prime}\right]-1 / 2 \mid$.

Definition 1 (INDr-sID-CPA). A depth $\lambda H I B E$ system $\mathcal{E}$ is selective-identity indistinguishable from random if for any probabilistic polynomial time (PPT) INDr-sID-CPA adversary $\mathcal{A}$, the function $\operatorname{Adv}^{\mathrm{HIBE}, \lambda, \mathcal{A}}(n)$ is negligible. We say that $\mathcal{E}$ is secure for the depth $\lambda$.

\subsection{Lattices and Gaussian Distributions}

For positive integers $n, m, q$ and a matrix $\mathbf{A} \in \mathbb{Z}^{n \times m}$, We consider lattices $\Lambda_{q}^{\perp}(\mathbf{A})=\left\{\mathbf{z} \in \mathbb{Z}^{m}: \mathbf{A} \mathbf{z}=\mathbf{0}(\bmod q)\right\} \Lambda_{q}^{\mathbf{u}}(\mathbf{A})=\left\{\mathbf{z} \in \mathbb{Z}^{m}: \mathbf{A z}=\mathbf{u}(\bmod q)\right.$. If $\Lambda_{q}^{\mathbf{u}}(\mathbf{A}) \neq \emptyset$ then $\Lambda_{q}^{\mathbf{u}}(\mathbf{A})$ is a shift of $\Lambda_{q}^{\perp}(\mathbf{A})$. Specifically, if there exists e such that $\mathbf{A e}=\mathbf{u}(\bmod q)$ then $\Lambda_{q}^{\mathbf{u}}(\mathbf{A})=\Lambda_{q}^{\perp}(\mathbf{A})+\mathbf{e}$.

Definition 2 (Gaussian Distribution). Given countable set $S \subset \mathbb{R}^{n}$ and $\sigma>0$, the discrete Gaussian distribution $D_{S, \sigma, \mathbf{c}}$ over $S$ centered at some $\mathbf{c} \in$ 
$S$ with standard deviation $\sigma$ is defined as $\mathcal{D}_{S, \sigma, \mathbf{c}}(\mathbf{x}):=\rho_{\sigma, \mathbf{c}}(\mathbf{x}) / \rho_{\sigma, \mathbf{c}}(S)$, where $\rho_{\sigma, \mathbf{c}}(\mathbf{x}):=\exp \left(\frac{-\pi\|\mathbf{x}-\mathbf{v}\|^{2}}{\sigma^{2}}\right)$ and $\rho_{\sigma, \mathbf{c}}(S):=\sum_{\mathbf{x} \in S} \rho_{\sigma, \mathbf{c}}(\mathbf{x})$. If $\mathbf{c}=\mathbf{0}$, we simply write $\rho_{\sigma}$ and $D_{S, \sigma}$ instead of $\rho_{\sigma, \mathbf{0}}, D_{S, \mathbf{0}, \sigma}$, respectively.

We use of the following tail bound of $D_{\Lambda, \sigma}$ for parameter $\sigma$ sufficiently larger than the smothing parameter $\eta_{\epsilon}(\Lambda)$, defined to be the the smallest real number $s$ such that $\rho_{1 / s}\left(\Lambda^{*} \backslash\{0\}\right) \leq \epsilon$; cf. [14].

Lemma 1 ( [6, Lemma 2.9]). For any $\epsilon>0$, any $\sigma \geq \eta_{\epsilon}(\mathbb{Z})$, and any $K>0$, we have $\operatorname{Pr}_{x \leftarrow D_{\mathbb{Z}, \sigma, c}}[|x-c| \geq K \cdot \sigma] \leq 2 e^{-\pi K^{2}} \cdot \frac{1+\epsilon}{1-\epsilon}$. In particular, if $\epsilon \in\left(0, \frac{1}{2}\right)$ and $K \geq \omega(\sqrt{\log n})$, then the probability that $|x-c| \geq K \cdot \sigma$ is negligible in $n$.

\subsection{Degree-Parametrized Middle-Product Learning with Errors}

Definition 3 (Middle-Product, [16, Definition 3.1]). Let $d_{a}, d_{b}, k, d$ be integers such that $d_{a}+d_{b}-1=2 k+d$. We define the middle-product of two polynomials $a \in \mathbb{Z}^{<d_{a}}[x]$ and $b \in \mathbb{Z}^{<d_{b}}[x]$ as follows:

$$
\odot_{d}: \mathbb{Z}^{<d_{a}}[x] \times \mathbb{Z}^{<d_{b}}[x] \rightarrow \mathbb{Z}^{<d}[x],(a, b) \mapsto\left\lfloor\frac{a b \bmod x^{k+d}}{x^{k}}\right\rfloor .
$$

Lemma 2 ( [16, Lemma 3.3]). Let $d, k, n>0$. For all $r \in R^{<k+1}[x], a \in$ $R^{<n}[x], s \in R^{<n+d+k-1}[x]$, it holds that $r \odot_{d}\left(a \odot_{d+k} s\right)=(r \cdot a) \odot_{d} s$.

Definition 4 (DMPLWE, [10, Definition 9]). Let $n^{\prime}>0, q \geq 2$, d = $\left(d_{1}, \cdots, d_{t^{\prime}}\right) \in\left[\frac{n^{\prime}}{2}\right]^{t^{\prime}}$, and let $\chi$ be a distribution over $\mathbb{R}_{q}$. For $s \in \mathbb{Z}_{q}^{<n^{\prime}-1}[x]$, we define the distribution $\operatorname{DMP}_{q, n^{\prime}, \mathbf{d}, \chi}(s)$ over $\prod_{i=1}^{t^{\prime}}\left(\mathbb{Z}_{q}^{n^{\prime}-d_{i}}[x] \times \mathbb{R}_{q}^{d_{i}}[x]\right)$ as follows:

- For each $i \in\left[t^{\prime}\right]$, sample $f_{i} \stackrel{\$}{\leftarrow} \mathbb{Z}_{q}^{<n^{\prime}-d_{i}}[x]$ and sample $e_{i} \leftarrow \chi^{d_{i}}[x]$ (represented as a polynomial of degree less than $\left.d_{i}\right)$.

- Output $\left(f_{i}, \mathrm{ct}_{i}:=f_{i} \odot_{d_{i}} s+e_{i}\right)_{i \in\left[t^{\prime}\right]}$.

The degree-parametrized MPLWE (named $\mathrm{DMPLWE}_{q, n^{\prime}, \boldsymbol{d}, \chi}$ ) requires to distinguish between arbitrarily many samples from $\operatorname{DMP}_{q, n^{\prime}, \mathbf{d}, \chi}(s)$ and the same number of samples from $\prod_{i=1}^{t^{\prime}} \mathcal{U}\left(\mathbb{Z}_{q}^{n^{\prime}-d_{i}}[x] \times \mathbb{R}_{q}^{d_{i}}[x]\right)$.

For $\mathcal{S}>0$, let $\mathcal{F}\left(\mathcal{S}, \mathbf{d}, n^{\prime}\right)$ be the set of monic polynomials $f$ in $\mathbb{Z}[x]$ with the constant coeficient coprime with $q$, that have degree $m \in \cap_{i=1}^{t^{\prime}}\left[d_{i}, n^{\prime}-d_{i}\right]$ and satisfy $\operatorname{EF}(f)<\mathcal{S}$. For a polynomial $f \in \mathbb{Z}[x]$ of degree $m, \operatorname{EF}(f)$ is the expansion factor ( [11]) of $f$ defined as follows: $\operatorname{EF}(f):=\max _{g \in \mathbb{Z}^{<2 m-1}[x]} \frac{\|g \bmod f\|_{\infty}}{\|g\|_{\infty}}$. Following [16], Lombardi et al. [10] showed that DMPLWE is as hard as $\operatorname{PLWE}_{q, \chi}^{(f)}$ (defined below) for any polynomial $f$ of poly $\left(n^{\prime}\right)$-bounded expansion factor.

Definition 5 (PLWE, [18]). Let $n>0, q \geq 2, f$ be a polynomial of degree $m$, $\chi$ be a distribution over $\mathbb{R}[x] / f$. The decision problem $\operatorname{PLWE}_{q, \chi}^{(f)}(s)$ is to distinguish between arbitrarily many samples $\left\{(a, a \cdot s+e): a \stackrel{\$}{\leftarrow} \mathbb{Z}_{q}[x] / f, e \leftarrow \chi\right\}$, and the same number of samples from $\mathcal{U}\left(\mathbb{Z}_{q}[x] / f \times \mathbb{R}_{q}[x] / f\right)$ over the randomness of $s \stackrel{\$}{\leftarrow} \mathbb{Z}_{q}[x] / f$. 
It is proven that $\operatorname{PLWE}_{q, \chi}^{(f)}(s)$ is as hard as solving Shortest Vector Problem (SVP) over ideal lattices in $\mathbb{Z}[x] / f$; see [18] for more detail.

Theorem 1 (Hardness of DMPLWE, [10, Theorem 2]). Let $n^{\prime}>0$, $q \geq 2, \mathbf{d}=\left(d_{1}, \cdots, d_{t^{\prime}}\right) \in\left[\frac{n^{\prime}}{2}\right]^{t^{\prime}}$, and $\alpha \in(0,1)$. Then, there exists a probabilistic polynomial time (PPT) reduction from $\operatorname{PLWE}_{q, D_{\alpha \cdot q}}^{(f)}$ for any polynomial $f$ in $\mathcal{F}\left(\mathcal{S}, \mathbf{d}, n^{\prime}\right)$ to $\operatorname{DMPLWE}_{q, n^{\prime}, \boldsymbol{d}, D_{\alpha^{\prime} \cdot q}}$ with $\alpha^{\prime}=\alpha \mathcal{S} \sqrt{\frac{n^{\prime}}{2}}$.

\subsection{Lattice Trapdoor Generation for DMPLWE}

Definition 6 (G-Trapdoor, [13, Definition 5.2]). Let $\mathbf{A} \in \mathbb{Z}_{q}^{n \times m}$ and $\mathbf{G} \in$ $\mathbb{Z}_{q}^{n \times m^{\prime}}$ be matrices with $m \geq m^{\prime} \geq n$. A matrix $\mathbf{R} \in \mathbb{Z}^{\left(m-m^{\prime}\right) \times m^{\prime}}$ is called $\mathbf{G}$ trapdoor for $\mathbf{A}$ with tag $\mathbf{H}$ (which is an invertible matrix in $\mathbb{Z}_{q}^{n \times n}$ ) if $\mathbf{A} \cdot\left[\begin{array}{c}\mathbf{R} \\ \mathbf{I}_{m^{\prime}}\end{array}\right]=$ HG.

In particular, it is suggested in [13, Section 4] that $\mathbf{G}=\mathbf{I}_{n} \otimes\left[\begin{array}{lll}1 & 2 \cdots 2^{k}\end{array}\right]$. We can choose $\mathbf{H}=\mathbf{I}_{n}$ or such that $\mathbf{H G}$ is any (column) permutation of $\mathbf{G}$ which is similar to the usage of $\mathbf{G}$ in [10]. In fact, it is defined in [10, Definition 11]) that $\mathbf{A} \in \mathbb{Z}^{k \times(m+k \tau)}$ and $\mathbf{G}:=\mathbf{I}_{k} \otimes\left[\begin{array}{lll}1 & 2 \cdots 2^{\tau-1}\end{array}\right] \in \mathbb{Z}_{q}^{k \times k \tau}$. However, $\mathbf{G}$ is used in SamplePre (see below) is actually a (column) permutation of $\mathbf{I}_{k} \otimes\left[12 \cdots 2^{\tau-1}\right]$ from which the authors can extracts polynomial $g_{i}$ in $\mathbf{g}$ thanks to the Toeplitz representation of polynomials (see Equation (6)). We first recall their definition and some basic properties.

Definition 7 (Toeplitz matrix). Let $R$ be a ring and $d, k>0$ be integers. For any polynomial $u \in R^{<n}[x]$, we define the Topelitz matrix $\operatorname{Tp}^{n, d}(u)$ for $u$ as a matrix in $R^{(n+d-1) \times d}$ whose the $i$-th column is the coefficient vector of $x^{i-1} \cdot u$ arranged in increasing degree of $x$ with 0 inserted if any.

By Definition 7, it is easy to assert the following Lemma.

Lemma 3. Let $u \in \mathbb{Z}^{<n}[x]$. Then,

$$
\operatorname{Tp}^{n, d}(u)=\left[\operatorname{Tp}^{n+d-1,1}(u)\left|\operatorname{Tp}^{n+d-1,1}(x \cdot u)\right| \cdots \mid \operatorname{Tp}^{n+d-1,1}\left(x^{d-1} \cdot u\right)\right] .
$$

Lemma 4 ( $[\mathbf{1 0}$, Lemma 7]). For positive integers $k, n, d$ and polynomials $u \in R^{<k}[x]$, if $v \in R^{<n}[x]$, then $\operatorname{Tp}^{k, n+d-1}(u) \cdot \operatorname{Tp}^{n, d}(v)=\operatorname{Tp}^{k+n-1, d}(u \cdot v)$.

Theorem 2 ( $\left[\mathbf{1 0 ,}\right.$ Theorem 4]). Let $\mathbf{G}:=\mathbf{I}_{k} \otimes\left[12 \cdots 2^{\tau-1}\right] \in \mathbb{Z}_{q}^{k \times k \tau}$ and matrices $\mathbf{A} \in \mathbb{Z}^{k \times(m+k \tau)}, \mathbf{R} \in \mathbb{Z}^{m \times k \tau}$ be such that $\mathbf{A} \cdot\left[\begin{array}{c}\mathbf{R} \\ \mathbf{I}_{k \tau}\end{array}\right]=\mathbf{G}$. Then, there exists an efficient algorithm $\mathcal{P}=\left(\mathcal{P}_{1}, \mathcal{P}_{2}\right)$ that executes according to the two following phases:

- offline: $\mathcal{P}_{1}(\mathbf{A}, \mathbf{R}, \sigma)$ performs some polynomial-time preprocessing on input $(\mathbf{A}, \mathbf{R}, \sigma)$ and outputs a state st. 
- online: for a given vector $\mathbf{u}, \mathcal{P}_{2}(\mathrm{st}, \mathbf{u})$ samples a vector from $D_{\Lambda_{u}^{\perp}(\boldsymbol{A}), \sigma}$ as long as

$$
\sigma \geq \omega(\sqrt{\log k}) \cdot \sqrt{7\left(s_{1}(\mathbf{R})^{2}+1\right)},
$$

where $s_{1}(\mathbf{R}):=\max _{\|\mathbf{u}\|=1}\|\mathbf{R u}\|$ is the largest singular value of $\mathbf{R}$.

The value $s_{1}(\mathbf{R})$ is upper bounded as explained by the lemma given below.

Lemma 5 ( $\left[\mathbf{1 0}\right.$, Lemma 6]). For any matrix $\mathbf{R}=\left(R_{i j}\right) \in \mathbb{R}^{m \times n}$,

$$
s_{1}(\mathbf{R}) \leq \sqrt{m n} \cdot \max _{i, j}\left|R_{i j}\right| .
$$

G-Trapdoor for a family of polynomials. We recap the construction of lattice trapdoors for DMPLWE from [10]. The construction applies two PPT algorithms TrapGen and SamplePre. Suppose that $q=\operatorname{poly}(n), d \leq n, d t / n=$ $\Omega(\log n), d \gamma=n+2 d-2, \tau:=\left\lceil\log _{2} q\right\rceil, \beta:=\left\lceil\frac{\log _{2}(n)}{2}\right\rceil \ll q / 2$, and $\sigma$ satisfies Equation (7) below. Then, TrapGen and SamplePre work as follows:

$\operatorname{TrapGen}\left(1^{n}\right)$ : On input a security parameter $n$, do the following:

- Sample $\overline{\mathbf{a}}^{\prime}=\left(a_{1}, \cdots, a_{t}\right) \stackrel{\$}{\leftarrow}\left(\mathbb{Z}_{q}^{<n}[x]\right)^{t}$, and for all $j \in[\gamma \tau]$, sample $\overline{\mathbf{w}}^{(j)}=\left(w_{1}^{(j)}, \cdots, w_{t}^{(j)}\right) \leftarrow\left(\Gamma^{d}[x]\right)^{t}$ where $\Gamma=\mathcal{U}(\{-\beta, \cdots, \beta\})$.

- For all $j \in[\gamma \tau]$, define $u_{j}=\left\langle\overline{\mathbf{a}}^{\prime}, \overline{\mathbf{w}}^{(j)}\right\rangle$ and $a_{t+j}=g_{j}-u_{j}$, where

$$
g_{j}=2^{\eta} x^{d \zeta} \in \mathbb{Z}_{q}^{n+d-1}[x],
$$

for $j=\zeta \tau+\eta+1$ with $\eta \in\{0, \cdots, \tau-1\}, \zeta \in\{0, \cdots, \gamma-1\}$. Set $\overline{\mathrm{g}}:=\left(g_{1}, \cdots, g_{\gamma \tau}\right)$.

- Output $\overline{\mathbf{a}}:=\left(a_{1}, \cdots, a_{t}, a_{t+1}, \cdots, a_{t+\gamma \tau}\right)$ with its corresponding trapdoor td $:=\left(\overline{\mathbf{w}}^{(1)}, \cdots, \overline{\mathbf{w}}^{(\gamma \tau)}\right)$.

The amount of space to store the trapdoor td is $O(d(\gamma \tau) t)=O(n \tau t)$ as $d \gamma=n+2 d-2 \leq 3 n$.

SamplePre $\left(\overline{\mathbf{a}}=\left(a_{1}, \cdots, a_{t+\gamma \tau}\right), \mathrm{td}=\left(\overline{\mathbf{w}}^{(1)}, \cdots, \overline{\mathbf{w}}^{(\gamma \tau)}\right), u, \sigma\right)$ : On input a family $\overline{\mathbf{a}}$ of $t+\gamma \tau$ polynomials together with its trapdoor $\mathrm{td}_{\epsilon}$ generated by TrapGen, and a polynomial $u$ of degree less than $n+2 d-2$, do the following:

- First, construct (implicitly) matrices $\mathbf{A}^{\prime}, \mathbf{A}, \mathbf{T}, \mathbf{G}$ for $\overline{\mathbf{a}}^{\prime}, \overline{\mathbf{a}}, \mathrm{td}, \overline{\mathbf{g}}$, respectively:

$$
\begin{gathered}
\mathbf{A}^{\prime}=\left[\mathrm{Tp}^{n, 2 d-1}\left(a_{1}\right)|\cdots| \mathrm{Tp}^{n, 2 d-1}\left(a_{t}\right)\right], \\
\mathbf{A}=\left[\mathrm{Tp}^{n, 2 d-1}\left(a_{1}\right)|\cdots| \mathrm{Tp}^{n, 2 d-1}\left(a_{t}\right)\left|\mathrm{Tp}^{n+d-1, d}\left(a_{t+1}\right)\right| \cdots \mid \mathrm{Tp}^{n+d-1, d}\left(a_{t+\gamma \tau}\right)\right], \\
\mathbf{T}=\left[\begin{array}{c}
\mathrm{Tp}^{d, d}\left(w_{1}^{(1)}\right) \cdots \mathrm{Tp}^{d, d}\left(w_{1}^{(\gamma \tau)}\right) \\
\vdots \\
\operatorname{Tp}^{d, d}\left(w_{t}^{(1)}\right) \cdots \mathrm{Tp}^{d, d}\left(w_{t}^{(\gamma \tau)}\right)
\end{array}\right] \in \mathbb{Z}_{q}^{(2 d-1) t \times d \gamma \tau} \\
\mathbf{G}=\left[\mathrm{Tp}^{n+d-1, d}\left(g_{1}\right)|\cdots| \mathrm{Tp}^{n+d-1, d}\left(g_{\gamma \tau}\right)\right] \in \mathbb{Z}_{q}^{d \gamma \times d \gamma \tau}
\end{gathered}
$$




$$
\mathbf{I}_{d \gamma \tau}=\left[\begin{array}{ccc}
\operatorname{Tp}^{1, d}(1) & \cdots & \\
\cdots & & \cdots \\
& \cdots & \operatorname{Tp}^{1, d}(1)
\end{array}\right] \in \mathbb{Z}_{q}^{d \gamma \tau \times d \gamma \tau} .
$$

Then $\mathbf{A}=\left[\mathbf{A}^{\prime} \mid \mathbf{G}-\mathbf{A}^{\prime} \mathbf{T}\right]$ and hence $\mathbf{A} \cdot\left[\begin{array}{c}\mathbf{T} \\ \mathbf{I}_{d \gamma \tau}\end{array}\right]=\mathbf{G}$. Recall that $d \gamma=$ $n+2 d-2$.

- The polynomial $u$ is represented it as $\mathbf{u}=\operatorname{Tp}^{n+2 d-2,1}(u) \in \mathbb{Z}_{q}^{n+2 d-2}$.

- Sample vector $\mathbf{r} \in \mathbb{Z}^{(2 d-1) t+d \gamma \tau}$ from $D_{\Lambda_{\mathbf{u}}^{\perp}(\mathbf{A}), \sigma}$ using the trapdoor $\mathbf{T}$ in means of [13], where

$$
\sigma \geq \omega(\sqrt{\log (d \gamma)}) \cdot \sqrt{7\left(s_{1}(\mathbf{T})^{2}+1\right)},
$$

and

$$
s_{1}(\mathbf{T}) \leq \sqrt{(2 d-1) t \cdot(d \gamma \tau)} \cdot \beta .
$$

- Split $\mathbf{r}$ into $\mathbf{r}=\left[\mathbf{r}_{1}^{\top}|\cdots| \mathbf{r}_{t+\gamma \tau}^{\top}\right]^{\top}$, and rewrite it (in column) as a Toeplitz matrix of polynomials $r_{1}, \cdots, r_{t+\gamma \tau}$, where $\mathbf{r}_{j}=\operatorname{Tp}^{2 d-1,1}\left(r_{j}\right), \operatorname{deg}\left(r_{j}\right)<$ $2 d-1, \forall j \in[t], \mathbf{r}_{j}=\operatorname{Tp}^{d, 1}\left(r_{j}\right), \operatorname{deg}\left(r_{t+j}\right)<d, \forall j \in t+1, \cdots, t+\gamma \tau$.

- Output $\overline{\mathbf{r}}:=\left(r_{1}, \cdots, r_{t+\gamma \tau}\right)$. Note that, $\langle\overline{\mathbf{a}}, \overline{\mathbf{r}}\rangle=\sum_{i=1}^{t+\gamma \tau} a_{i} \cdot r_{i}=u$; see [10, Section 5] for more details.

The runtime of SamplePre is $\tilde{O}(n t)$ and the output distribution of $\left(r_{i}\right)$ is exactly the conditional distribution

$$
\left(D_{\mathbb{Z}^{2 d-1}, \sigma}[x]\right)^{t} \times\left(D_{\mathbb{Z}^{d}, \sigma}[x]\right)^{\gamma \tau} \mid \sum_{i=1}^{t+\gamma \tau} a_{i} \cdot r_{i}=u .
$$

Further on, we give our main results, which are a trapdoor delegation mechanism useful for extending a family of polynomials as well as a HIBE system built using the framework of [1]. From now on, by "trapdoor", we mean "G-trapdoor", where $\mathbf{G}$ is defined by Equation (6). Also, we denote the output of TrapGen by $\overline{\mathbf{a}}_{\epsilon}$ and $\mathrm{td}_{\epsilon}$ and call them the root family and the root trapdoor, respectively. The Toeplitz matrices $\mathbf{A}_{\epsilon}$ and $\mathbf{T}_{\epsilon}$ correspond to $\overline{\mathbf{a}}_{\epsilon}$ and $\mathrm{td}_{\epsilon}$, respectively.

\section{Trapdoor Delegation for Polynomials}

\subsection{Description}

In order to exploit the trapdoor technique in constructing a MPLWE-based HIBE scheme, we have to solve the problem of delegating a trapdoor (in the sense of Definition 6) for $\overline{\mathbf{f}}=\left(a_{1}, \cdots, a_{t^{\prime}} \mid h_{1}, \cdots, h_{t^{\prime \prime}}\right)$ provided the trapdoor for $\overline{\mathbf{a}}=\left(a_{1}, \cdots, a_{t^{\prime}}\right)$. As mentioned in Section 2.4, we can represent $\overline{\mathbf{f}}$ as a concatenation of Toeplitz matrices of the form $\mathbf{F}=[\mathbf{A} \mid \mathbf{H}]$ in which $\mathbf{A}, \mathbf{H}$ are the Toeplitz representations for $\overline{\mathbf{a}}$ and $\overline{\mathbf{h}}:=\left(h_{1}, \cdots, h_{t^{\prime \prime}}\right)$, respectively.

Following Definition 6, our task is to find a matrix $\mathbf{R}$, which satisfies the equation $\mathbf{F} \cdot\left[\begin{array}{c}\mathbf{R} \\ \mathbf{I}\end{array}\right]=\mathbf{G}$, where $\mathbf{G}$ as given by Equation (6). Recall that, in matrix 
setting in [13, Section 5.5], this task can be easily done by finding $\mathbf{R}$ that satisfies the relation $\mathbf{A R}=\mathbf{G}-\mathbf{H}$, when we know a trapdoor for $\mathbf{A}$ and $\mathbf{H}$ has the same dimension as $\mathbf{G}$. In our setting, this task is not straightforward. The main reason for this is that the matrices $\mathbf{A}, \mathbf{G}, \mathbf{H}$ are Toeplitz ones. To be able to apply the idea of trapdoor delegation of [13] to our setting, we have to design $\mathbf{H}$ such that $\mathbf{U}:=\mathbf{G}-\mathbf{H}$ is still in the Toeplitz form of some polynomials. In other words, the form of $\mathbf{H}$ should be similar in form and in dimension to that of $\mathbf{G}$ in (6), namely,

$$
\mathbf{H}=\left[\mathrm{Tp}^{n+d-1, d}\left(h_{1}\right)|\cdots| \mathrm{Tp}^{n+d-1, d}\left(h_{\gamma \tau}\right)\right] \in \mathbb{Z}_{q}^{d \gamma \times d \gamma \tau} .
$$

This requires that $t^{\prime \prime}=\gamma \tau$ and $\operatorname{deg}\left(h_{i}\right)<n+d-1$ for all $i \in[\gamma \tau]$. If this is the case, the last step is to try to follow [10] using SamplePre to have $\mathbf{R}$ satisfy $\mathbf{A R}=\mathbf{U}$ given $\mathbf{A}$ and a trapdoor for $\mathbf{A}$. Note that in our polynomial setting $\mathbf{R}$ should be a structured matrix, which can be easily converted into appropriate polynomials $r_{i}$.

By generalization, we come up with the following theorem in which $t^{\prime}=$ $t+k \gamma \tau$ and $t^{\prime \prime}=m \gamma \tau$ for $k \geq 1, m \geq 1$ :

Theorem 3 (Trapdoor Delegation). Let $n$ be a positive integer, $q=\operatorname{poly}(n)$ be a prime, and $d, t, \gamma, \tau, k, m$ be positive integers such that $d \leq n, d t / n=$ $\Omega(\log n), d \gamma=n+2 d-2, k \geq 1, m \geq 1$. Let $\tau:=\left\lceil\log _{2} q\right\rceil$ and $\beta:=\left\lceil\frac{\log _{2} n}{2}\right\rceil$. Let $\mathbf{G}$ be matrix as in (6) and $\overline{\mathbf{a}}=\left(a_{1}, \cdots, a_{t+k \gamma \tau}\right)$ be $a(t+k \gamma \tau)$-family of polynomials and its associated trapdoor $\operatorname{td}_{a}$, where $a_{i} \in \mathbb{Z}_{q}^{<n}[x]$ for $i \in[t]$ and $a_{i} \in \mathbb{Z}_{q}^{<n+d-1}[x]$ for $t+1 \leq i \leq t+k \gamma \tau$. Suppose that $\overline{\mathbf{h}}=\left(h_{1}, \cdots, h_{m \gamma \tau}\right)$ is a $m \gamma \tau$-family of polynomials in $\mathbb{Z}_{q}^{<n+d-1}[x]$ and $\bar{\sigma}=\left(\sigma_{k+1}, \cdots, \sigma_{k+m}\right)$ to be determined. Then, there exists an efficient (PPT) algorithm, SampleTrap $\left(\overline{\mathbf{a}}, \overline{\mathbf{h}}, \mathrm{td}_{a}, \bar{\sigma}\right)$ that outputs a trapdoor $\operatorname{td}_{f}$ for $\overline{\mathbf{f}}=\left(a_{1}, \cdots, a_{t+k \gamma \tau} \mid h_{1}, \cdots, h_{m \gamma \tau}\right)$. Moreover, the amount of space to store the trapdoor $\operatorname{td}_{f}$ is $O(((2 d-1) t+(k+m-1) \gamma \tau) \cdot d \gamma \tau)=$ $O\left(n^{2} \log ^{2} n\right)=\widetilde{O}\left(n^{2}\right)$.

\subsection{Elementary Trapdoor Delegation}

In this section, we present in detail the basic trapdoor delegation for the family $\overline{\mathbf{f}}=\left(a_{1}, \cdots, a_{t+\gamma \tau} \mid h_{1}, \cdots, h_{\gamma \tau}\right)$ given the root trapdoor $\mathrm{td}_{\epsilon}$ for the root family $\overline{\mathbf{a}}_{\epsilon}=\left(a_{1}, \cdots, a_{t+\gamma \tau}\right)$. They are generated by TrapGen, i.e., SampleTrap for $k=1$ and $m=1$. This process is called TrapDel and is shown as Algorithm 1 .

Note that TrapGen, $\overline{\mathbf{a}}_{\epsilon}=\left(a_{1}, \cdots, a_{t}, a_{t+1}, \cdots, a_{t+\gamma \tau}\right) \in\left(\mathbb{Z}_{q}^{<n}[x]\right)^{t} \times\left(\mathbb{Z}_{q}^{<n+d-1}[x]\right)^{\gamma \tau}$, and the corresponding concatenated Toeplitz matrix $\mathbf{A}_{\epsilon} \in \mathbb{Z}_{q}^{(n+2 d-2) \times[(2 d-1) t+d \gamma \tau]}$ is constructed as

$$
\mathbf{A}_{\epsilon}=\left[\mathrm{Tp}^{n, 2 d-1}\left(a_{1}\right)|\cdots| \mathrm{Tp}^{n, 2 d-1}\left(a_{t}\right)\left|\mathrm{Tp}^{n+d-1, d}\left(a_{t+1}\right)\right| \cdots \mid \mathrm{Tp}^{n+d-1, d}\left(a_{t+\gamma \tau}\right)\right] .
$$

The matrix $\mathbf{G}$ has the following form:

$$
\mathbf{G}=\left[\operatorname{Tp}^{n+d-1, d}\left(g_{1}\right)|\cdots| \mathrm{Tp}^{n+d-1, d}\left(g_{\gamma \tau}\right)\right]
$$


where $g_{j}=2^{\eta} x^{d \zeta}$ for $j=\zeta \tau+\eta+1$ with $\eta \in\{0, \cdots, \tau-1\}, \zeta \in\{0, \cdots, \gamma-1\}$. As discussed above, we construct $\mathbf{H}=\left[\mathrm{Tp}^{n+d-1, d}\left(h_{1}\right)|\cdots| \mathrm{Tp}^{n+d-1, d}\left(h_{\gamma \tau}\right)\right]$ for $h_{1}, \cdots, h_{\gamma \tau}$, whose $\operatorname{deg}\left(h_{i}\right)<n+d-1$ for all $i \in[\gamma \tau]$. Then the Toeplitz matrix for $\overline{\mathbf{f}}$ takes the form

$$
\begin{aligned}
\mathbf{F} & =\left[\mathbf{A}_{\epsilon} \mid \mathbf{H}\right] \\
& =\left[\mathrm{Tp}^{n, 2 d-1}\left(a_{1}\right)|\cdots| \mathrm{Tp}^{n, 2 d-1}\left(a_{t}\right)\left|\mathrm{Tp}^{n+d-1, d}\left(a_{t+1}\right)\right| \cdots \mid \mathrm{Tp}^{n+d-1, d}\left(h_{\gamma \tau}\right)\right] .
\end{aligned}
$$

and

$$
\mathbf{G}-\mathbf{H}=\left[\mathrm{Tp}^{n+d-1, d}\left(g_{1}-h_{1}\right)|\cdots| \mathrm{Tp}^{n+d-1, d}\left(g_{\gamma \tau}-h_{\gamma \tau}\right)\right] .
$$

For $i=1, \cdots, \gamma \tau$, let $u_{i}=g_{i}-h_{i}$. From Lemma 3, we have

$$
\begin{aligned}
\mathbf{G}-\mathbf{H}=[ & {\left[\operatorname{Tp}^{n+2 d-2,1}\left(u_{1}\right)|\cdots| \operatorname{Tp}^{n+2 d-2,1}\left(x^{d-1} \cdot\left(u_{1}\right)\right) \mid\right.} \\
& \left.\cdots\left|\operatorname{Tp}^{n+2 d-2,1}\left(u_{\gamma \tau}\right)\right| \cdots \mid \operatorname{Tp}^{n+2 d-2,1}\left(x^{d-1} \cdot\left(u_{\gamma \tau}\right)\right)\right] \\
= & {\left[\operatorname{Tp}^{n+2 d-2,1}\left(v_{1}\right)|\cdots| \operatorname{Tp}^{n+2 d-2,1}\left(x^{d-1} \cdot\left(v_{d \gamma \tau}\right)\right)\right], }
\end{aligned}
$$

where $v_{i}=x^{\alpha} u_{\beta}$ for $i=\alpha+d(\beta-1)+1$, with $\alpha \in\{0, \cdots, d-1\}, \beta \in$ $\{1, \cdots, \gamma \tau\}$. Let $\mathbf{v}^{(i)}:=\operatorname{Tp}^{n+2 d-2,1}\left(v_{i}\right)$. Now, for $i=1, \cdots, \gamma \tau$ we have to find $\mathbf{R}=\left[\mathbf{r}^{(1)}|\cdots| \mathbf{r}^{(d \gamma \tau)}\right]$ such that $\mathbf{A}_{\epsilon}\left[\mathbf{r}^{(1)}|\cdots| \mathbf{r}^{(d \gamma \tau)}\right]=\left[\mathbf{v}^{(1)}|\cdots| \mathbf{v}^{(d \gamma \tau)}\right]$, which is equivalent to $\mathbf{A}_{\epsilon} \mathbf{r}^{(i)}=\mathbf{v}^{(i)}$ for $1 \leq i \leq d \gamma \tau$. This can be done using SamplePre $\left(\overline{\mathbf{a}}_{\epsilon}\right.$, $\left.\operatorname{td}_{\epsilon}, v_{i}, \sigma\right)$. Eventually, we get $\mathbf{r}^{(i)} \in \mathbb{Z}^{(2 d-1) t+d \gamma \tau}$, which is sampled from $\mathcal{D}_{\Lambda_{\mathbf{v}^{(i)}}(\mathbf{A}), \sigma}$, where $\sigma \geq \omega(\sqrt{\log (d \gamma)}) \cdot \sqrt{7\left((2 d-1) t \cdot(d \gamma \tau) \cdot \beta^{2}+1\right)}$; see $(7),(8)$.

Finally, we obtain the trapdoor $\operatorname{td}_{f}=\left(\overline{\mathbf{r}}^{(1)}, \cdots, \overline{\mathbf{r}}^{(d \gamma \tau)}\right)$ for $\overline{\mathbf{f}}$, where $\overline{\mathbf{r}}^{(i)}=$ $\left(r_{1}^{(i)}, \cdots, r_{t+\gamma \tau}^{(i)}\right)$, with $\operatorname{deg}\left(r_{j}^{(i)}\right)<2 d-1$ for $j \in[t], \operatorname{deg}\left(r_{t+j}^{(i)}\right)<d$ for $j \in[\gamma \tau]$ and for all $i \in[d \gamma \tau]$. and its corresponding matrix representation is

$$
\mathbf{R}=\left(R_{i j}\right)=\left[\begin{array}{ccc}
\operatorname{Tp}^{2 d-1,1}\left(r_{1}^{(1)}\right) & \cdots & \operatorname{Tp}^{2 d-1,1}\left(r_{1}^{(d \gamma \tau)}\right) \\
\vdots & & \vdots \\
\operatorname{Tp}^{2 d-1,1}\left(r_{t}^{(1)}\right) & \cdots & \operatorname{Tp}^{2 d-1,1}\left(r_{t}^{(d \gamma \tau)}\right) \\
\operatorname{Tp}^{d, 1}\left(r_{t+1}^{(1)}\right) & \cdots & \operatorname{Tp}^{d, 1}\left(r_{t+1}^{(d \gamma \tau)}\right) \\
\vdots & & \vdots \\
\operatorname{Tp}^{d, 1}\left(r_{t+\gamma \tau}^{(1)}\right) & \cdots & \operatorname{Tp}^{d, 1}\left(r_{t+\gamma \tau}^{(d \gamma \tau)}\right)
\end{array}\right] \in \mathbb{Z}^{((2 d-1) t+d \gamma \tau) \times d \gamma \tau}
$$

Certainly, we have $\mathbf{F} \cdot\left[\begin{array}{c}\mathbf{R} \\ \mathbf{I}\end{array}\right]=\mathbf{G}$. Remark that, by Lemma 1,

$$
\left|R_{i j}\right| \leq \omega(\log n) \cdot \sigma \text { with probability } 1-\operatorname{neg}(n) .
$$

Hence, from Lemma 5

$$
s_{1}(\mathbf{R}) \leq \sqrt{((2 d-1) t+d \gamma \tau) \cdot(d \gamma \tau)} \cdot \omega(\log n) \cdot \sigma,
$$

where $\sigma$ satisfies Equation (7). 


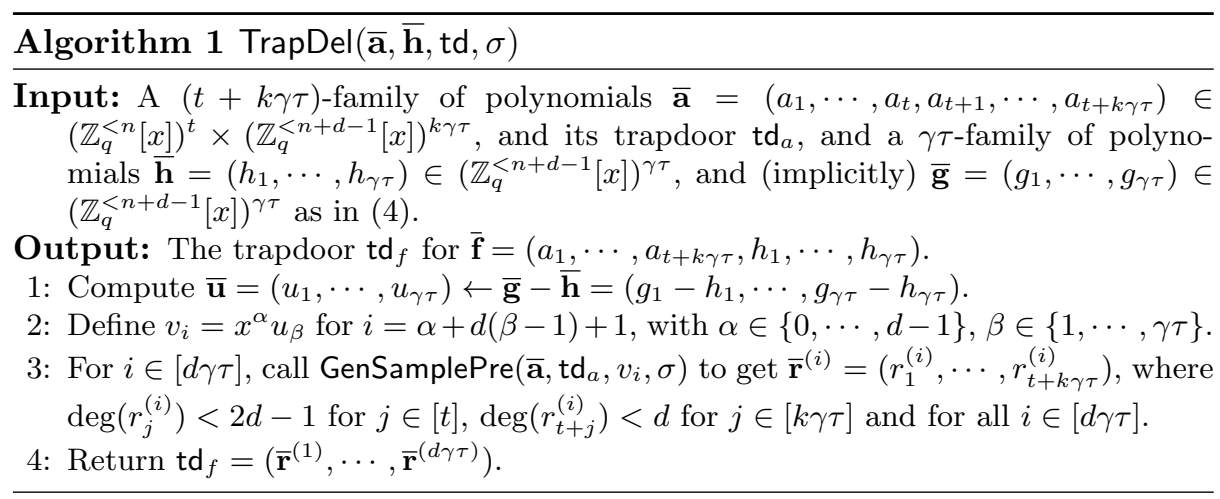

Note that, after having the trapdoor for $\overline{\mathbf{f}}$ and by assigning $\overline{\mathbf{a}}_{\epsilon} \leftarrow \overline{\mathbf{f}}, \mathbf{A}_{\epsilon} \leftarrow \mathbf{F}$, we can perform the same procedure explained above. So we get a trapdoor for $\overline{\mathbf{f}}^{\prime}=\left(a_{1}, \cdots, a_{t+\gamma \tau}, h_{1}, \cdots, h_{\gamma \tau} \mid z_{1}, \cdots, z_{\gamma \tau}\right)$ for some $\overline{\mathbf{z}}=\left(z_{1}, \cdots, z_{\gamma \tau}\right)$, where $z_{i} \in \mathbb{Z}_{q}^{<n+d-1}[x]$. Consequently, we come up with a PPT algorithm called TrapDel (Algorithm 1) in which we consider the expanded families of the form $\overline{\mathbf{a}}=$ $\left(a_{1}, \cdots, a_{t}, a_{t+1}, \cdots, a_{t+k \gamma \tau}\right) \in\left(\mathbb{Z}_{q}^{<n}[x]\right)^{t} \times\left(\mathbb{Z}_{q}^{<n+d-1}[x]\right)^{k \gamma \tau}$ for $k \geq 1$. Also note that TrapDel does not call SamplePre. Instead, it calls a slightly modified variant presented below.

Generalized SamplePre. Accordingly to the expansion of trapdoors, we slightly modify SamplePre in Section 2.4 and call it GenSamplePre. The algorithm works not only with TrapGen (i.e., $k=1$ ) but also with TrapDel (i.e., $k>1$ ). GenSamplePre is the same as SamplePre except for $k>1$, where matrices $\mathbf{R}$ given as the input trapdoors are of form (13), while for $k=1$, the matrix $\mathbf{R}$ is of form (5). If we execute GenSamplePre for input $\left(\overline{\mathbf{a}}=\left(a_{1}, \cdots, a_{t+k \gamma \tau}\right), \operatorname{td}_{a}=\right.$ $\left.\left(\overline{\mathbf{r}}^{(1)}, \cdots, \overline{\mathbf{r}}^{(d \gamma \tau)}\right), u, \sigma\right)$, where $\overline{\mathbf{r}}^{(i)}=\left(r_{1}^{(i)}, \cdots, r_{k \gamma \tau}^{(i)}\right)($ with $k>1)$, then $\operatorname{td}_{a}$ should be interpreted as a $((2 d-1) t+(k-1) d \gamma \tau) \times d \gamma \tau$-matrix, say $\mathbf{R}^{(k-1)}$, of the form (13). The last row is indexed by $t+(k-1) \gamma \tau$.

\subsection{SampleTrap}

SampleTrap mentioned in Theorem 3 is described as follows:

SampleTrap $\left(\overline{\mathbf{a}}=\left(a_{1}, \cdots, a_{t+k \gamma \tau}\right), \overline{\mathbf{h}}=\left(h_{1}, \cdots, h_{m \gamma \tau}\right), \operatorname{td}_{a}, \bar{\sigma}=\left(\sigma_{k+1}, \cdots, \sigma_{k+m}\right)\right)$ :

- Input: A $(t+k \gamma \tau)$-family of polynomials $\overline{\mathbf{a}}=\left(a_{1}, \cdots, a_{t}, a_{t+1}, \cdots, a_{t+k \gamma \tau}\right) \in$ $\left(\mathbb{Z}_{q}^{<n}[x]\right)^{t} \times\left(\mathbb{Z}_{q}^{<n+d-1}[x]\right)^{k \gamma \tau}$, its trapdoor $\mathrm{td}_{a}$ and a $m \gamma \tau$-family of polynomials $\overline{\mathbf{h}}=\left(h_{0}, \cdots, h_{m \gamma \tau}\right) \in\left(\mathbb{Z}_{q}^{<n+d-1}[x]\right)^{m \gamma \tau}$, where $m \geq 1$, and (implicitly) $\overline{\mathbf{g}}=\left(g_{1}, \cdots, g_{\gamma \tau}\right) \in\left(\mathbb{Z}_{q}^{<n+d-1}[x]\right)^{\gamma \tau}$ as in (4).

- Output: The trapdoor $\operatorname{td}_{f}$ for $\overline{\mathbf{f}}=\left(a_{1}, \cdots, a_{t+k \gamma \tau} \mid h_{1}, \cdots, h_{m \gamma \tau}\right)$.

- Execution:

1. Split $\overline{\mathbf{h}}=\left(\overline{\mathbf{h}}^{(1)}, \cdots, \overline{\mathbf{h}}^{(m)}\right)$ where each $\overline{\mathbf{h}}^{(i)}$ is a $\gamma \tau$-family of polynomials. 
2. $\mathrm{td}^{(1)} \leftarrow \mathrm{td}_{a}, \overline{\mathbf{a}}^{(1)} \leftarrow \overline{\mathbf{a}}$.

3. For $i=1$ up to $m$ do:

- $\mathrm{td}^{(i+1)} \leftarrow \operatorname{TrapDel}\left(\overline{\mathbf{a}}^{(i)}, \overline{\mathbf{h}}^{(i)}, \mathrm{td}^{(i)}, \sigma_{i}\right)$.

- $\overline{\mathbf{a}}^{(i+1)} \leftarrow\left(\overline{\mathbf{a}}^{(i)}, \overline{\mathbf{h}}^{(i)}\right)$.

4. Return $\operatorname{td}_{f}=\operatorname{td}^{(m+1)}$.

Let us make few observations for SampleTrap.

Trapdoor $\mathbf{t} \mathbf{d}_{\boldsymbol{f}}$. From Section 3.2, we can easily generalize to see that the output $\operatorname{td}_{f}$ is $\left(\overline{\mathbf{r}}^{(1)}, \cdots, \overline{\mathbf{r}}^{(d \gamma \tau)}\right)$ in which for $i \in[d \gamma \tau], \overline{\mathbf{r}}^{(i)}=\left(r_{1}^{(i)}, \cdots, r_{t+(k+m-1) \gamma \tau}^{(i)}\right)$ and $r_{j}^{(i)} \in \mathbb{Z}_{q}^{<n+d-1}[x]$ for $j \in[t]$, and $r_{t+j}^{(i)} \in \mathbb{Z}_{q}^{<d}[x]$ for $j \in[(k+m-1) \gamma \tau]$. We can imply that the matrix representation, named $\mathbf{R}^{(k+m-1)}$, for the trapdoor $\operatorname{td}_{f}$ has the form (13), with the last row's index $t+(k+m-1) \gamma \tau$.

Setting Gaussian parameters $\bar{\sigma}=\left(\sigma_{1}, \cdots, \sigma_{m}\right)$. Note that the algorithm SamplePre $\left(\overline{\mathbf{a}}, \operatorname{td}_{a}, u, \bar{\sigma}\right)$ has to satisfy Condition (7) for each $\sigma_{i}$. The same condition must hold for GenSamplePre. From Equation (13), we can see that the trapdoor $\mathrm{td}^{(i+1)}$ in SampleTrap can be interpreted as a matrix $\mathbf{R}^{(i+1)}$ of dimension $((2 d-1) t+(k+i-1) d \gamma \tau) \times(d \gamma \tau)$. Thus, $\sigma_{i}$ in Equations (14) and (15) should satisfy $\sigma_{i} \geq \omega(\sqrt{\log (d \gamma)}) \cdot \sqrt{7\left(s_{1}\left(\mathbf{R}^{(i-1)}\right)^{2}+1\right)}$, and

$s_{1}\left(\mathbf{R}^{(i-1)}\right) \leq \sqrt{((2 d-1) t+(k+i-1) d \gamma \tau) \cdot(d \gamma \tau)} \cdot \omega(\log n) \cdot \sigma_{i-1}$, where $i \in[m]$.

\section{DMPLWE-based HIBE in Standard Model}

In this section, we describe a HIBE system based on the DMPLWE problem. Our HIBE scheme is IND-sID-CPA secure in the standard model and is inspired by the construction of IBE from [1]. Note that the authors of [10] use a similar approach. However, the private key $\mathrm{SK}_{\text {id }}$ (with respect to an identity id) in the standard model IBE of [10] is actually not a trapdoor. Therefore, it seems difficult to construct HIBE using this approach. In our HIBE construction, the private key for an identity id $=\left(i d_{1}, \cdots, i d_{\ell}\right)$ of depth $\ell$ is a trapdoor for a family of polynomials, which corresponds to the public key. So we can derive the private key for the appended identity id $\mid i d_{k}=\left(i d_{1}, \cdots, i d_{\ell}, \cdots i d_{k}\right)$ using the trapdoor delegation presented in Section 3, where $k>\ell$.

\subsection{Construction}

Our construction, named HIBE, consists of a tuple of algorithms \{Setup, Extract, Derive, Encrypt, Decrypt\}. They are described below.

$\operatorname{Setup}\left(1^{\lambda}, 1^{n}\right)$ : On input the security parameter $n$, the maximum depth $\lambda$, perform the following: 
- Set common parameters as follows:

- $q=q(n)$ be a prime; $d, k$ be positive integers such that $2 d+k \leq n$ and $\frac{n+2 d-2}{d}$ is also a positive integer, say $\gamma$, i.e., $d \gamma=n+2 d-2$; $\beta:=\left\lceil\frac{\log _{2} n}{2}\right\rceil, \tau:=\left\lceil\log _{2} q\right\rceil, t$ is a positive integer and let $t^{\prime}=t+\gamma \tau$, and plaintext space $\mathcal{M}:=\{0,1\}^{<k+2}[x]$.. Note that we will set $t^{\prime}=$ $m \gamma \tau$ (with $m \geq 2$ ), that is $t$ is a multiple of $\gamma \tau$ so as to we can apply the trapdoor delegation.

- For Gaussian parameters used in Encrypt: choose $\bar{\alpha}=\left(\alpha_{1}, \cdots, \alpha_{\lambda}\right) \in$ $\mathbb{R}_{>0}^{\lambda}$; for Gaussian parameters used in Extract and Derive: choose $\bar{\Sigma}=$ $\left(\bar{\sigma}^{(1)}, \cdots, \bar{\sigma}^{(\lambda)}\right)$, where $\bar{\sigma}^{(\ell)}=\left(\sigma_{1}^{(\ell)}, \cdots, \sigma_{m}^{(\ell)}\right) \in \mathbb{R}_{>0}^{m}$. For $\ell \in[\lambda]$, let $\bar{\Sigma}^{(\ell)}=\left(\bar{\sigma}^{(1)}, \cdots, \bar{\sigma}^{(\ell)}\right)$; for Gaussian parameters used in Decrypt: choose $\bar{\Psi}=\left(\Psi_{1}, \cdots, \Psi_{\lambda}\right) \in \mathbb{R}_{>0}^{\lambda}$.

They all are set as in Section 4.2.

- For $\ell \in[\lambda]$, let $\chi_{\ell}:=\left\lfloor D_{\alpha_{\ell} \cdot q}\right\rceil$ be the rounded Gaussian distribution.

- Use TrapGen $\left(1^{n}\right)$ to get a root family $\overline{\mathbf{a}}_{\epsilon}=\left(a_{1}, \cdots, a_{t^{\prime}}\right)$ and its associated root trapdoor $\mathrm{td}_{\epsilon}$.

- Select uniformly a random polynomial $u_{0} \in \mathbb{Z}_{q}^{<n+2 d-2}[x]$.

- For each $i \in[\lambda]$, and each bit $\in\{0,1\}$, sample randomly $\overline{\mathbf{h}}^{(i, \mathrm{bit})}=$ $\left(h_{1}^{(i, \text { bit })}, \cdots, h_{t^{\prime}}^{(i, \text { bit })}\right.$, where each $h_{j}^{(i, \text { bit })} \in \mathbb{Z}_{q}^{<n}[x]$ for $j \in[t]$, and each $h_{j}^{(i, \text { bit })} \in \mathbb{Z}_{q}^{<n+d-1}[x]$ for $j \in\{t+1, \cdots, t+\gamma \tau\}$. Let HList $=\left\{\left(i\right.\right.$, bit, $\left.\overline{\mathbf{h}}^{(i, \text { bit })}\right):$ $i \in[\lambda]$, bit $\in\{0,1\}\}$ be the ordered set of all $\overline{\mathbf{h}}^{(i, \text { bit })}$.

- Set the master secret key MSK $:=\mathrm{td}_{\epsilon}$.

We denote id $=\left(i d_{1}, \cdots, i d_{\ell}\right) \in\{0,1\}^{\ell}$ as an identity of depth $\ell \leq \lambda$. All following algorithms will always work on $\overline{\mathbf{a}}_{\epsilon}=\left(a_{1}, \cdots, a_{t^{\prime}}\right)$ and HList.

Derive $\left(\mathrm{id} \mid i d_{\ell+1}, \mathrm{SK}_{\mathrm{id}}\right):$ On input id $=\left(i d_{1}, \cdots, i d_{\ell}\right), \mathrm{id} \mid i d_{\ell+1}=\left(i d_{1}, \cdots, i d_{\ell}, i d_{\ell+1}\right)$, private key $\mathrm{SK}_{\mathrm{id}}:=\mathrm{td}_{\mathrm{id}}-$ the trapdoor for $\overline{\mathbf{f}}_{\mathrm{id}}=\left(\overline{\mathbf{a}}_{\epsilon}, \overline{\mathbf{h}}^{\left(1, i d_{1}\right)}, \ldots, \overline{\mathbf{h}}^{\left(\ell, i d_{\ell}\right)}\right)$, execute:

1. Output $\mathrm{SK}_{\mathrm{id} \mid i d_{\ell+1}} \leftarrow$ SampleTrap $\left(\overline{\mathbf{f}}_{\mathrm{id}}, \overline{\mathbf{h}}^{\left(\ell+1, i d_{\ell+1}\right)}, \mathrm{SK}_{\mathrm{id}}, \bar{\Sigma}^{(\ell+1)}\right)$.

Extract(id, MSK): On input id $=\left(i d_{1}, \cdots, i d_{\ell}\right), \mathrm{MSK}=\mathrm{td}_{\epsilon}$, execute:

1. Build $\overline{\mathbf{h}}_{\text {id }}=\left(\overline{\mathbf{h}}^{\left(1, i d_{1}\right)}, \cdots, \overline{\mathbf{h}}^{\left(\ell, i d_{\ell}\right)}\right)$.

2. Output $\mathrm{SK}_{\text {id }} \leftarrow$ SampleTrap $\left(\overline{\mathbf{a}}_{\epsilon}, \overline{\mathbf{h}}_{\text {id }}, \mathrm{MSK}, \bar{\Sigma}^{(\ell)}\right)$.

Encrypt $\left(\mathrm{id}, \mu, u_{0}, \alpha_{\ell}\right)$ : On input id $=\left(i d_{1}, \cdots, i d_{\ell}\right), \mu \in \mathcal{M}, u_{0}, \alpha_{\ell}$, execute:

1. Build $\left(f_{1}, \cdots, f_{t^{\prime}(\ell+1)}\right) \leftarrow \overline{\mathbf{f}}_{\mathrm{id}}=\left(\overline{\mathbf{a}}_{\epsilon}, \overline{\mathbf{h}}^{\left(1, i d_{1}\right)} \cdots, \overline{\mathbf{h}}^{\left(\ell, i d_{\ell}\right)}\right)$.

2. Sample $s \stackrel{\$}{\leftarrow} \mathbb{Z}_{q}^{<n+2 d+k-1}[x]$.

3. Sample $e_{0} \leftarrow \chi_{\ell}^{k+1}[x]$, compute: $\mathrm{CT}_{0}=u_{0} \odot_{k+2} s+2 e_{0}+\mu$.

4. For $i=0$ to $\ell$ do:

- For $j \in[t]$, sample $e_{i \cdot t^{\prime}+j} \leftarrow \chi_{\ell}^{2 d+k}[x]$, and compute:

$$
\mathrm{ct}_{i}=f_{i \cdot t^{\prime}+j} \odot_{2 d+k} s+2 e_{i \cdot t^{\prime}+j} .
$$

- For $t+1 \leq j \leq t+\gamma \tau$, sample $e_{i \cdot t^{\prime}+j} \leftarrow \chi_{\ell}^{d+k+1}[x]$, and compute:

$$
\mathrm{ct}_{i}=f_{i \cdot t^{\prime}+j} \odot_{d+k+1} s+2 e_{i \cdot t^{\prime}+j} .
$$


5. Set $\mathrm{CT}_{1}=\left(\mathrm{ct}_{1}, \cdots, \mathrm{ct}_{t^{\prime}(\ell+1)}\right)$, and output ciphertext $\overline{\mathrm{CT}}=\left(\mathrm{CT}_{0}, \mathrm{CT}_{1}\right)$. Decrypt $\left(\mathrm{id}, \mathrm{SK}_{\mathrm{id}}, \overline{\mathrm{CT}}, u_{0}, \Psi_{\ell}\right)$ : On input id $=\left(i d_{1}, \cdots, i d_{\ell}\right), \mathrm{SK}_{\text {id }}:=\mathrm{td}_{\mathrm{id}}-$ the trapdoor for $\overline{\mathbf{f}}_{\text {id }}=\left(\overline{\mathbf{a}}_{\epsilon}, \overline{\mathbf{h}}^{\left(1, i d_{1}\right)}, \cdots, \overline{\mathbf{h}}^{\left(\ell, i d_{\ell}\right)}\right)$, ciphertext $\overline{\mathrm{CT}}=\left(\mathrm{CT}_{0}, \mathrm{CT}_{1}\right)$, $u_{0}$, and $\Psi_{\ell}$, do:

1. Parse $\left(f_{1}, \cdots, f_{t^{\prime}(\ell+1)}\right) \leftarrow \overline{\mathbf{f}}_{\text {id }}=\left(\overline{\mathbf{a}}_{\epsilon}, \overline{\mathbf{h}}^{\left(1, i d_{1}\right)}, \cdots, \overline{\mathbf{h}}^{\left(\ell, i d_{\ell}\right)}\right)$.

2. Sample $\overline{\mathbf{r}}=\left(r_{1}, \cdots, r_{t^{\prime}(\ell+1)}\right) \leftarrow$ GenSamplePre $\left(\overline{\mathbf{f}}_{\text {id }}, \mathrm{SK}_{\text {id }}, u_{0}, \Psi_{\ell}\right)$, i.e., $\left\langle\overline{\mathbf{f}}_{\text {id }}, \overline{\mathbf{r}}\right\rangle=\sum_{1}^{t^{\prime}(\ell+1)} r_{i} \cdot f_{i}=u_{0}$.

3. Parse $\left(\mathrm{CT}_{0}, \mathrm{CT}_{1}=\left(\mathrm{ct}_{1}, \cdots, \mathrm{ct}_{t^{\prime}(\ell+1)}\right)\right) \leftarrow \overline{\mathrm{CT}}$.

4. Output $\mu=\left(\mathrm{CT}_{0}-\sum_{i=1}^{t^{\prime}(\ell+1)} \mathrm{ct}_{i} \odot_{k+2} r_{i} \bmod q\right) \bmod 2$.

\subsection{Correctness and Parameters}

Lemma 6 (Correctness). For $\ell \in[\lambda]$, if

$$
\alpha_{\ell}<\frac{1}{4}\left[t^{\prime}(\ell+1) \cdot(k+1) \cdot \omega(\log n) \cdot \Psi_{\ell}+\omega(\sqrt{\log n})\right]^{-1},
$$

then the scheme is correct with probability $1-\operatorname{negl}(n)$.

Proof. For id $=\left(i d_{1}, \cdots, i d_{\ell}\right)$, we need to show that

$$
\left.\operatorname{Decrypt}\left(\mathrm{id}, \mathrm{SK}_{\text {id }} \text {, Encrypt(id, } \mu, u_{0}, \alpha_{\ell}\right), u_{0}, \Psi_{\ell}\right)=\mu,
$$

with probability $1-\operatorname{neg}(n)$ over the randomness of Setup, Derive, Extract, Encrypt. Suppose that $\overline{\mathrm{CT}}:=\left(\mathrm{CT}_{0}, \mathrm{CT}_{1}=\left(\mathrm{ct}_{1}, \cdots, \mathrm{ct}_{t^{\prime}(\ell+1)}\right)\right) \leftarrow$ Encrypt $\left(\mathrm{id}, \mu, u_{0}, \alpha_{\ell}\right)$. By Lemma 2, we have

$$
\mathrm{CT}_{0}-\sum_{i=1}^{t^{\prime}(\ell+1)} \mathrm{ct}_{i} \odot_{k+2} r_{i}=\mu+2\left(e_{0}-\sum_{1}^{t^{\prime}(\ell+1)} r_{i} \odot_{k+2} e_{i}\right) .
$$

Hence, if $\left\|\mu+2\left(e_{0}-\sum_{1}^{t^{\prime}(\ell+1)} r_{i} \odot_{k+2} e_{i}\right)\right\|_{\infty}<q / 2$ then $\mu$ is recovered.

Therefore, we need to bound the coefficients of $e_{0}-\sum_{1}^{t^{\prime}(\ell+1)} r_{i} \odot_{k+2} e_{i}$. First, note that,

- for $i \in[t]: \operatorname{deg}\left(r_{i}\right)<d_{r}:=2 d-1, \operatorname{deg}\left(e_{i}\right)<d_{e}:=k+1$.

- for $i \in\left\{t+1, \cdots, t^{\prime}(\ell+1)\right\}: \operatorname{deg}\left(r_{i}\right)<d_{r}:=d, \operatorname{deg}\left(e_{i}\right)<d_{e}:=d+k+1$.

In gerneral, $d_{e}+d_{r}-1=2(d-1)+(k+2)$. Let $\mathbf{r}_{i}=\left(\mathbf{r}_{i, 0}, \cdots, \mathbf{r}_{i, d_{r}-1}\right), \mathbf{e}_{i}=$ $\left(\mathbf{e}_{i, 0}, \cdots, \mathbf{e}_{i, d_{e}-1}\right)$ be the vectors of coefficients of $r_{i}$ and $e_{i}$, respectively. By definition of the middle product, $r_{i} \odot_{k+2} e_{i}=\sum_{j+w=d-1}^{d+k} \mathbf{r}_{i, j} \cdot \mathbf{e}_{i, w} \cdot x^{j+w}$. By Lemma $1, \operatorname{Pr}\left[\left\|\mathbf{r}_{i}\right\|_{\infty}>\omega(\sqrt{\log n}) \cdot \Psi_{\ell}\right]=\operatorname{neg} \mid(n), \operatorname{Pr}\left[\left\|\mathbf{e}_{i}\right\|_{\infty}>\omega(\sqrt{\log n}) \cdot \alpha_{\ell} \cdot q\right]=$ $\operatorname{neg}(n)$.

Hence $\left\|r_{i} \odot_{k+2} e_{i}\right\|_{\infty}<(k+2) \cdot \omega(\log n) \cdot \Psi_{\ell} \cdot \alpha_{\ell} \cdot q$. As a result,

$$
\left\|e_{0}-\sum_{1}^{t^{\prime}(\ell+1)} r_{i} \odot_{k+2} e_{i}\right\|_{\infty} \leq\left[t^{\prime}(\ell+1) \cdot(k+2) \cdot \omega(\log n) \cdot \Psi_{\ell}+\omega(\sqrt{\log n})\right] \cdot \alpha_{\ell} \cdot q .
$$

In order for the decryption to be correct, we need Condition (16). 
Setting Parameters. We set the parameters as described below:

- Security prameter $n, q=\operatorname{poly}(n)$ prime, $\beta:=\left\lceil\frac{\log _{2}(n)}{2}\right\rceil \ll q / 2, \tau:=\left\lceil\log _{2}(q)\right\rceil$, $\tau=\Theta(\log q)=\Theta(\log n), t^{\prime}=t+\gamma \tau=m \gamma \tau$ (for some $\left.m \geq 2\right), d \leq n$, $d t / n=\Omega(\log n)$, and $d \gamma=n+2 d-2 \leq 3 n$.

- We set Gaussian parameters used in Extract and Derive as follows: Recall that, for $\ell \in[\lambda], \bar{\Sigma}^{(\ell)}=\left(\bar{\sigma}^{(1)}, \cdots, \bar{\sigma}^{(\ell)}\right)$, where each $\bar{\sigma}^{(i)}=\left(\sigma_{1}^{(i)}, \cdots, \sigma_{m}^{(i)}\right) \in$ $\mathbb{R}_{>0}^{m}$. It suffices to consider the maximal case happening in Extract in which $\bar{\Sigma}=\left(\bar{\sigma}^{(1)}, \cdots, \bar{\sigma}^{(\lambda)}\right)$. Now, we renumber $\bar{\Sigma}$ as $\left(\sigma_{1}, \cdots, \sigma_{m \lambda}\right)$ without changing their order. For the maximal identity id $=\left(i d_{1}, \cdots, i d_{\lambda}\right)$, we build $\overline{\mathbf{h}}_{\text {id }}=\left(\overline{\mathbf{h}}^{\left(1, i d_{1}\right)}, \cdots, \overline{\mathbf{h}}^{\left(\lambda, i d_{\lambda}\right)}\right)$ and then compute $\mathrm{SK}_{\text {id }}$ by calling SampleTrap for input $\left(\overline{\mathbf{a}}_{\epsilon}, \overline{\mathbf{h}}_{\text {id }}\right.$, MSK, $\left.\bar{\Sigma}\right)$. We now split $\overline{\mathbf{h}}_{\text {id }}$ into $\left(\overline{\mathbf{h}}^{(1)}, \cdots, \overline{\mathbf{h}}^{(m \lambda)}\right)$ and let $\overline{\mathbf{a}}^{(i)}=$ $\left(\overline{\mathbf{a}}_{\epsilon}\left|\overline{\mathbf{h}}^{(1)}\right| \cdots \mid \overline{\mathbf{h}}^{(i)}\right)$ with $\overline{\mathbf{a}}^{(0)}=\overline{\mathbf{a}}_{\epsilon}$. Then, SampleTrap calls TrapDel $\left(\overline{\mathbf{a}}^{(i-1)}, \overline{\mathbf{h}}^{(i)}\right.$, $\left.\mathrm{td}^{(i-1)}, \sigma_{i}\right)$ up to $m \lambda$ times for $i \in[m \lambda]$, in which $\mathrm{td}^{(0)}=\mathrm{td}_{\epsilon}$ and $\mathrm{td}^{(i-1)}$ is the output of the previous execution of $\operatorname{TrapDel}\left(\overline{\mathbf{a}}^{(i-2)}, \overline{\mathbf{h}}^{(i-1)}, \mathrm{td}^{(i-2)}, \sigma_{i-1}\right)$, for $2 \leq i \leq m \lambda$. Now, all $\sigma_{i}$ 's are set in the same way as in Section 3.3, that is, for $2 \leq i \leq m \lambda, \sigma_{i} \geq \omega(\sqrt{\log (d \gamma)}) \cdot \sqrt{7\left(s_{1}\left(\mathbf{R}^{(i-1)}\right)^{2}+1\right)}$, and

$$
s_{1}\left(\mathbf{R}^{(i-1)}\right) \leq \sqrt{((2 d-1) t+(i-1) d \gamma \tau) \cdot(d \gamma \tau)} \cdot \omega(\log n) \cdot \sigma_{i-1},
$$

in which $\mathbf{R}^{(i-1)}$ is the matrix representation, as in (13) with the last row's in$\operatorname{dex} t+(i-1) \gamma \tau$, of the private key (the trapdoor) for $\overline{\mathbf{a}}^{(i-1)}=\left(\overline{\mathbf{a}}_{\epsilon}\left|\overline{\mathbf{h}}^{(1)}\right| \cdots \mid \overline{\mathbf{h}}^{(i-1)}\right)$, with $\sigma_{1}$ and $\mathbf{R}^{(1)}$ play the role of $\sigma$ and $\mathbf{T}$ in (7), (8).

- We set Gaussian parameters used in Decrypt $\bar{\Psi}=\left(\Psi_{1}, \cdots, \Psi_{\lambda}\right)$ as follows: For $\ell \in[\lambda]$, since $\Psi_{\ell}$ is used in GenSamplePre $\left(\overline{\mathbf{f}}_{\text {id }}, \mathrm{SK}_{\text {id }}, u_{0}, \Psi_{\ell}\right)$ with $\mathrm{SK}_{\text {id }}=\operatorname{td}_{\text {id }}$ the trapdoor for $\overline{\mathbf{f}}_{\text {id }}=\left(\overline{\mathbf{a}}_{\epsilon}, \overline{\mathbf{h}}^{\left(1, i d_{1}\right)}, \cdots, \overline{\mathbf{h}}^{\left(\ell, i d_{\ell}\right)}\right)$ which equals to $\overline{\mathbf{a}}^{(\ell m)}$ above. Therefore, for $\ell \in[\lambda-1]$ we can set $\Psi_{\ell}=\sigma_{\ell m+1}$, and

$$
\begin{gathered}
\Psi_{\lambda} \geq \omega(\sqrt{\log (d \gamma)}) \cdot \sqrt{7\left(s_{1}\left(\mathbf{R}^{(m \lambda)}\right)^{2}+1\right)}, \\
s_{1}\left(\mathbf{R}^{(m \lambda)}\right) \leq \sqrt{((2 d-1) t+(m \lambda) d \gamma \tau) \cdot(d \gamma \tau)} \cdot \omega(\log n) \cdot \sigma_{m \lambda},
\end{gathered}
$$

in which $\mathbf{R}^{(m \lambda)}$ is the matrix representation for the private key (the trapdoor) for $\overline{\mathbf{a}}^{(m \lambda)}=\left(\overline{\mathbf{a}}_{\epsilon}\left|\overline{\mathbf{h}}^{(1)}\right| \cdots \mid \overline{\mathbf{h}}^{(m \lambda)}\right)$.

- We set Gaussian parameters used in Encrypt $\bar{\alpha}=\left(\alpha_{1}, \cdots, \alpha_{\lambda}\right)$ such that for $\ell \in[\lambda], \alpha_{\ell}$ satisfies (16).

\subsection{Security Analysis}

Theorem 4. The proposed HIBE system is INDr-sID-CPA secure in the standard model under the DMPLWE assumption.

Proof. We construct a sequence of games from $G_{0}$ to $G_{4}$ in which an INDr-sID-CPA adversary can distinguish two consecutive games $G_{i}$ and $G_{i+1}$ with negligible probability only. In particular, for the transition of the last two games $G_{3}$ and 
$G_{4}$, we show by contradiction that if there exists an adversary whose views are different in each game, i.e., the adversary can distinguish $G_{3}$ from $G_{4}$ with non-negligible probability, then we can build an adversary who can solve the underlying DMPLWE problem.

Game $G_{0}$ is the original IND-sID-CPA game between the adversary $\mathcal{A}$ and the $\overline{\text { challenger }} \mathcal{C}$. Note that, we are working with the selective game: at the beginning, $\mathcal{A}$ lets the challenger know the target identity $\mathrm{id}^{*}=\left(i d_{1}^{*}, \cdots, i d_{\theta}^{*}\right)$ that $\mathcal{A}$ intends to atack, where $\theta \leq \lambda$. Then, $\mathcal{C}$ runs Setup to choose randomly a vector of polynomials $\overline{\mathbf{a}}_{\epsilon}=\left(a_{1}, \cdots, a_{t^{\prime}}\right)$ together with an associated trapdoor $\mathrm{td}_{\epsilon}$, a set of polynomial vectors sampled randomly $\overline{\mathbf{h}}^{(i, \text { bit })}=\left(h_{1}^{(i, \text { bit })}, \cdots, h_{t^{\prime}}^{(i, \text { bit })}\right)$, which are stored in HList0, where each $h_{j}^{(i, \text { bit })} \in \mathbb{Z}_{q}^{<n}[x]$ for $j \in[t]$, and each $h_{j}^{(i, \text { bit })} \in \mathbb{Z}_{q}^{<n+d-1}[x]$ for $j \in\{t+1, \cdots, t+\gamma \tau\}$, and $\mathcal{C}$ also chooses a random polynomial $u_{0} \in \mathbb{Z}_{q}^{<n+2 d-2}[x]$. The challenger then sets MSK $:=\operatorname{td}_{\epsilon}$ as the master secret key. Furthermore, at the Challenge Phase, the challenger also generates a challenge ciphertext $\overline{\mathrm{CT}}^{*}$ for the identity $\mathrm{id}^{*}$.

Game $G_{1}$ is the same as $G_{0}$ except that in the Setup Phase the challenger $\overline{\mathcal{C} \text { generates }}\left(\overline{\mathbf{h}}^{(i, \text { bit })}\right)_{0 \leq i \leq \lambda, \text { bit } \in\{0,1\}}$ stored in HList1 $:=\left\{\left(i\right.\right.$, bit, $\left.\overline{\mathbf{h}}^{(i, \text { bit })}\right): i \in$ $[\lambda]$, bit $\in\{0,1\}\}$ with the corresponding trapdoor $\mathrm{td}^{(i, \text { bit })}$ stored in TList1 $:=$ $\left\{\left(i\right.\right.$, bit, td $\left.^{(i, \text { bit })}\right): i \in[\lambda]$, bit $\left.\in\{0,1\}\right\}$ using TrapGen.

Game $G_{2}$ is the same as $G_{1}$, except that the challenger $\mathcal{C}$ does not use $\operatorname{td}_{\epsilon}$ as the master secret key nor the Extract algorithm to response a private key queries on $\mathrm{id}=\left(i d_{1}, \cdots, i d_{\ell}\right)$ which is not a prefix of the target $\mathrm{id}^{*}$, where $\ell \leq \lambda$. Instead, $\mathcal{C}$ designs a new procedure TrapExtract with the knowledge of TList1. TrapExtract requires not all of TList1 but only one $\mathrm{td}^{\left(j, i d_{j}\right)} \in \mathrm{TList1}$ for any $j \in[\ell]$.

$\underline{\operatorname{TrapExtract}\left(\overline{\mathbf{a}}_{\epsilon}, \mathrm{HList} 1, \mathrm{id}=\left(i d_{1}, \cdots, i d_{\ell}\right), j, \mathrm{td}^{\left(j, i d_{j}\right)}\right):}$

1. Build $\overline{\mathbf{f}}_{\text {id }}=\left(\overline{\mathbf{a}}_{\epsilon}, \overline{\mathbf{h}}^{\left(1, i d_{1}\right)}, \ldots, \overline{\mathbf{h}}^{\left(j-1, i d_{j-1}\right)}, \overline{\mathbf{h}}^{\left(j+1, i d_{j+1}\right)}, \ldots, \overline{\mathbf{h}}^{\left(\ell, i d_{\ell}\right)}\right)$

2. $\mathrm{SK}_{\mathrm{id}} \leftarrow$ SampleTrap $\left(\overline{\mathbf{h}}^{\left(j, i d_{j}\right)}, \overline{\mathbf{f}}_{\mathrm{id}}, \mathrm{td}^{\left(j, i d_{j}\right)}, \bar{\Sigma}^{(\ell)}\right)$

Game $G_{3}$ is the same as $G_{2}$, except that in the Setup Phase, the challenger $\mathcal{C}$ generates HList3 as follows:

- For each $j \in[\lambda]$ and bit $\in\{0,1\}$ such that bit $\neq i d_{j}^{*}, \mathcal{C}$ calls TrapGen to generate $\overline{\mathbf{h}}^{(j, \text { bit })}$ and its associated trapdoor $\mathrm{td}^{(j, \text { bit })}$.

- For each $j \in[\lambda]$ and bit $\in\{0,1\}$ such that bit $=i d_{j}^{*}, \mathcal{C}$ simply samples $\overline{\mathbf{h}}^{(j, \text { bit })}$ uniformly at random and set $\mathrm{td}^{(j, \mathrm{bit})}=\perp$.

The challenger then put all $\overline{\mathbf{h}}^{(j, \text { bit })}$ into HList3 and all td ${ }^{(j, \text { bit })}$ into TList3. At the moment, to response a private key query on identity id $=\left(i d_{1}, \cdots, i d_{\ell}\right)$ which is not a prefix of the target identity id*, the challenger chooses an index $j^{\dagger}$ such that 
$i d_{j^{\dagger}} \neq i d_{j^{\dagger}}^{*}$. It then runs TrapExtract $\left(\overline{\mathbf{a}}_{\epsilon}\right.$, HList3,id $\left.=\left(i d_{1}, \cdots, i d_{\ell}\right), j^{\dagger}, \mathrm{td}^{\left(j^{\dagger}, i d_{j^{\dagger}}\right)}\right)$, where $\mathrm{td}^{\left(j^{\dagger}, i d_{j^{\dagger}}\right)} \in \mathrm{TList} 3$, and gives the result $\mathrm{SK}_{\mathrm{id}}$ to the adversary. At the Challenge Phase, the challenge ciphertext $\overline{\mathrm{CT}}^{*}$ is generated by computing Encrypt(id, $\left.\mu, u_{0}, \bar{\alpha}\right)$ over HList3.

Game $G_{4}$ is the same as $G_{3}$, except that the challenge ciphertext $\overline{\mathrm{CT}}^{*}=$ $\left(\mathrm{CT}_{0}^{*}, \mathrm{CT}_{1}^{*}\right)$ is chosen uniformly at random by the challenger.

In what follows, we show the indistinguishability of the games. It is easy to see that the view of the adversary is identical in games $G_{0}$ and $G_{1}$, in games $G_{1}$ and $G_{2}$, in games $G_{2}$ and $G_{3}$, except in games $G_{3}$ and $G_{4}$. We show that the view of the adversary is indistinguishable in these two games. We proceed by contradiction. Assume that the adversary $\mathcal{A}$ can distinguish between games $G_{3}$ and $G_{4}$ with non-negligile probability. Then we construct an adversary $\mathcal{B}$ that is able to solve DMPLWE problem with the same probability. The reduction from DMPLWE is as follows:

- Instance: Assume that the goal of $\mathcal{B}$ is to decide whether $1+t^{\prime}(\ell+1)$ samples $\left(f_{z}, \mathrm{ct}_{z}\right)$ for $z \in\left\{0, \cdots, t^{\prime}(\ell+1)\right\}$ (i) follow $\prod_{z=0}^{t^{\prime}(\ell+1)} \mathcal{U}\left(\mathbb{Z}_{q}^{n^{\prime}-d_{z}}[x] \times \mathbb{R}_{q}^{d_{z}[x]}\right)$, or (ii) follow $\operatorname{DMP}_{q, n^{\prime}, \mathbf{d}, \chi}(s)$, where $n^{\prime}=n+2 d+k$ and

- $\mathbf{d}=\left(d_{0}, d_{1}, \cdots, d_{t^{\prime}(\ell+1)}\right)$ is interpreted as follows: $d_{0}:=k+2$ and for $i \in\{0, \cdots, \ell\}, d_{i \cdot t^{\prime}+j}= \begin{cases}2 d+k, & \text { if } j \in[t], \\ d+k+1, & \text { if } j \in\{t+1, \cdots, t+\gamma \tau\} .\end{cases}$

- $f_{z}$ are random in $\mathbb{Z}_{q}^{<n^{\prime}-d_{z}}[x]$ for $z \in\left\{0, \cdots, t^{\prime}(\ell+1)\right\}$.

In other words, $\mathcal{B}$ has to distinguish whether (i) all $\mathrm{ct}_{z}$ are random or (ii) $\mathrm{ct}_{z}=f_{z} \odot_{d_{z}} s+2 e_{z}$ in $\mathbb{Z}_{q}^{<d_{z}}[x]$, for some $s \stackrel{\$}{\leftarrow} \mathbb{Z}_{q}^{<n^{\prime}-1}[x]$ and $e_{z} \leftarrow \chi^{d_{z}}[x]$, for all $z \in\left\{0, \cdots, t^{\prime}(\ell+1)\right\}$.

- Targeting: $\mathcal{B}$ receives from the adversary $\mathcal{A}$ the target identity id* that $\mathcal{A}$ wants to attack.

- Setup:. $\mathcal{B}$ generates HListB in the same way as in Game $G_{3}$ and Game $G_{4}$ as follows:

- For each $j \in[\lambda]$ and bit $\in\{0,1\}$ such that bit $\neq i d_{j}^{*}: \mathcal{B}$ calls TrapGen to generate $\overline{\mathbf{h}}^{(j, \text { bit })}$ and its associated trapdoor $\operatorname{td}^{(j, \text { bit })}$.

- For each $j \in[\lambda]$ and bit $\in\{0,1\}$ such that bit $=i d_{j}^{*}: \mathcal{B}$ simply samples $\overline{\mathbf{h}}^{(j, \text { bit })}$ uniformly at random and set $\mathrm{td}^{(j, \text { bit })}=\perp$.

The challenger then put all $\overline{\mathbf{h}}^{(j, \text { bit })}$ into HListB and all td ${ }^{(j, \text { bit })}$ into TListB.

- Queries: To response the private key queries, $\mathcal{B}$ acts as in Game $G_{3}$ or in Game $G_{4}$ using one of trapdoors that is not $\perp$.

- Challenge: To produce the challenge ciphertext, $\mathcal{B}$ chooses randomly $b \stackrel{\$}{\leftarrow}$ $\{0,1\}$ and sets $\overline{\mathrm{CT}}^{*}:=\left(\mathrm{CT}_{0}^{*}:=c t_{0}+\mu, \mathrm{CT}_{1}^{*}:=\left(\mathrm{ct}_{1}, \cdots, \mathrm{ct}_{t^{\prime}(\ell+1)}\right)\right)$.

- Guess: Eventually, $\mathcal{A}$ has to guess and output the value of $b$. Then, $\mathcal{B}$ returns what $\mathcal{A}$ outputted.

Analysis. Clearly, from the view of $\mathcal{A}$, the behaviour of $\mathcal{B}$ is almost identical in both Games $G_{3}$ and $G_{4}$. The only different thing is producing the challenge 
ciphertext. Specifically, if ct $_{z}$ 's are DMPLWE samples then the components of $\overline{\mathrm{CT}}^{*}$ are distributed as in Game $G_{3}$, while $\mathrm{ct}_{z}$ 's are random then the components of $\overline{\mathrm{CT}}^{*}$ are distributed as in Game $G_{4}$. Since $\mathcal{A}$ can distinguish between Games $G_{3}$ and $G_{4}$ with non-negligile probability, then so can $\mathcal{B}$ in solving DMPLWE with the same probability.

\section{Conclusions}

In this paper, we present a trapdoor delegation method that enables us to obtain a trapdoor for an expanded set of polynomials from a given trapdoor for a subset of the set. Also, thanks to the polynomial trapdoor delegation, we built a hierarchical identity-based encryption system that is secure in the standard model under the DMPLWE assumption.

Acknowledgment. We all would like to thank anonymous reviewers for their helpful comments. This work is partially supported by the Australian Research Council Discovery Project DP200100144. The first author has been sponsored by a Data61 PhD Scholarship. The fourth author has been supported by the Australian ARC grant DP180102199 and Polish NCN grant 2018/31/B/ST6/03003.

\section{References}

1. Agrawal, S., Boneh, D.: Identity-Based Encryption from Lattices in the Standard Model. In: Manuscript (2009), http://www.robotics.stanford.edu/ xb/ab09/ latticeibe.pdf

2. Agrawal, S., Boneh, D., Boyen, X.: Efficient Lattice (H)IBE in the Standard Model. In: Gilbert, H. (ed.) Advances in Cryptology - EUROCRYPT 2010. pp. 553-572. Springer Berlin Heidelberg, Berlin, Heidelberg (2010). https://doi.org/10.1007/978-3-642-13190-5_28

3. Canetti, R., Halevi, S., Katz, J.: A Forward-Secure Public-Key Encryption Scheme. In: Biham, E. (ed.) Advances in Cryptology — EUROCRYPT 2003. pp. 255-271. Springer Berlin Heidelberg, Berlin, Heidelberg (2003). https://doi.org/10.1007/3540-39200-9_16

4. Cash, D., Hofheinz, D., Kiltz, E., Peikert, C.: Bonsai Trees, or How to Delegate a Lattice Basis. In: Gilbert, H. (ed.) Advances in Cryptology - EUROCRYPT 2010. pp. 523-552. Springer Berlin Heidelberg, Berlin, Heidelberg (2010). https://doi.org/10.1007/978-3-642-13190-5_27

5. Dodis, Y., Fazio, N.: Public Key Broadcast Encryption for Stateless Receivers. In: Feigenbaum, J. (ed.) Digital Rights Management. pp. 61-80. Springer Berlin Heidelberg, Berlin, Heidelberg (2003). https://doi.org/0.1007/978-3-540-44993-5_5

6. Gentry, C., Peikert, C., Vaikuntanathan, V.: Trapdoors for Hard Lattices and New Cryptographic Constructions. In: Proceedings of the Fortieth Annual ACM Symposium on Theory of Computing. pp. 197-206. STOC '08, ACM, New York, NY, USA (2008). https://doi.org/10.1145/1374376.1374407

7. Gentry, C., Silverberg, A.: Hierarchical ID-Based Cryptography. In: Zheng, Y. (ed.) Advances in Cryptology - ASIACRYPT 2002. pp. 548-566. Springer Berlin Heidelberg, Berlin, Heidelberg (2002). https://doi.org/10.1007/3-540-36178-2_34 
8. Hengartner, U., Steenkiste, P.: Exploiting Hierarchical Identity-Based Encryption for Access Control to Pervasive Computing Information. In: First International Conference on Security and Privacy for Emerging Areas in Communications Networks (SECURECOMM'05). pp. 384-396 (2005). https://doi.org/10.1109/SECURECOMM.2005.18

9. Horwitz, J., Lynn, B.: Toward Hierarchical Identity-Based Encryption. In: Knudsen, L.R. (ed.) Advances in Cryptology - EUROCRYPT 2002. pp. 466-481. Springer Berlin Heidelberg, Berlin, Heidelberg (2002). https://doi.org/10.1007/3540-46035-7_31

10. Lombardi, A., Vaikuntanathan, V., Vuong, T.D.: Lattice Trapdoors and IBE from Middle-Product LWE. In: Hofheinz, D., Rosen, A. (eds.) Theory of Cryptography. pp. 24-54. Springer International Publishing, Cham (2019). https://doi.org/10.1007/978-3-030-36030-6_2

11. Lyubashevsky, V., Micciancio, D.: Generalized Compact Knapsacks Are Collision Resistant. In: Bugliesi, M., Preneel, B., Sassone, V., Wegener, I. (eds.) Automata, Languages and Programming. pp. 144-155. Springer Berlin Heidelberg, Berlin, Heidelberg (2006). https://doi.org/10.1007/11787006_13

12. Lyubashevsky, V., Peikert, C., Regev, O.: On ideal lattices and learning with errors over rings. In: Advances in Cryptology - EUROCRYPT 2010, 29th Annual International Conference on the Theory and Applications of Cryptographic Techniques, Monaco / French Riviera, May 30 - June 3, 2010. Proceedings. pp. 1-23 (2010). https://doi.org/10.1007/978-3-642-13190-5_1

13. Micciancio, D., Peikert, C.: Trapdoors for Lattices: Simpler, Tighter, Faster, Smaller. In: Proceedings of the 31st Annual International Conference on Theory and Applications of Cryptographic Techniques. pp. 700-718. EUROCRYPT'12, Springer-Verlag, Berlin, Heidelberg (2012). https://doi.org/10.1007/978-3-64229011-4_41

14. Micciancio, D., Regev, O.: Worst-Case to Average-Case Reductions Based on Gaussian Measures. SIAM J. Comput. 37(1), 267-302 (Apr 2007). https://doi.org/10.1137/S0097539705447360

15. Pan, V.Y.: Structured Matrices and Polynomials: Unified Superfast Algorithms. Springer-Verlag, Berlin, Heidelberg (2001)

16. Roşca, M., Sakzad, A., Stehlé, D., Steinfeld, R.: Middle-Product Learning with Errors. In: Katz, J., Shacham, H. (eds.) Advances in Cryptology CRYPTO 2017. pp. 283-297. Springer International Publishing, Cham (2017). https://doi.org/10.1007/978-3-319-63697-9_10

17. Shamir, A.: Identity-Based Cryptosystems and Signature Schemes. In: Blakley, G.R., Chaum, D. (eds.) Advances in Cryptology. pp. 47-53. Springer Berlin Heidelberg, Berlin, Heidelberg (1985). https://doi.org/10.1007/3-540-39568-7_5

18. Stehlé, D., Steinfeld, R., Tanaka, K., Xagawa, K.: Efficient public key encryption based on ideal lattices. In: Advances in Cryptology - ASIACRYPT 2009, 15th International Conference on the Theory and Application of Cryptology and Information Security, Tokyo, Japan, December 6-10, 2009. Proceedings. pp. 617-635 (2009). https://doi.org/10.1007/978-3-642-10366-7_36

19. Yao, D., Fazio, N., Dodis, Y., Lysyanskaya, A.: ID-Based Encryption for Complex Hierarchies with Applications to Forward Security and Broadcast Encryption. In: Proceedings of the 11th ACM Conference on Computer and Communications Security. p. 354-363. CCS '04, Association for Computing Machinery, New York, NY, USA (2004). https://doi.org/10.1145/1030083.1030130 